\title{
SUR LES ÉQUATIONS AUX DIFFÉRENCES FINIES.
}

PAR

\author{
M. GHERMANESCO
}

à BuCARest.

Les équations aux différences finies, connues du temps d'Euler, Bernoulli, Lagrange, etc. ont fait l'objet des préoccupations de nombre de mathématiciens, dont les efforts se sont concentrés surtout à l'étude de l'équation ${ }^{1}$

$$
f(x+1)-f(x)=g(x)
$$

qui en était la plus simple, du moins, quant à la forme.

Mais ces efforts n'ont pas porté tout leur fruit pour deux causes essentielles: la première, on ne pouvait pas s'assurer de l'uniforme convergence des développements des solutions, dont les polynomes de Bernoulli y étaient l'instrument analytique primordial et la deuxième, la solution de l'équation (A) dépendant d'une fonction arbitraire, on ne pouvait pas distinguer entre les propriétés qui caractérisaient toute solution de l'équation (A) et celles introduites par la fonction arbitraire considérée.

C'est plus tard que $\mathrm{N}$. Nielsen ${ }^{2}$ parvient à remédier partiellement au premier inconvenient en introduisant les polynomes $\varphi_{n}(x)$, ayant une étroite parenté avec ceux de Bernoulli et dont il détermine la valeur asymptotique, ce qui permet d'obtenir une solution absolument et uniformément convergente, lorsque $g(x)$ est une fonction entière de genre un au plus.

C. Guichard ${ }^{3}$, P. Appell ${ }^{4}$ et A. Hurwitz ${ }^{5}$ donnent une nouvelle impulsion

${ }^{1}$ Pour l'historique complet on peut lire l'article de M. NöRlund, "Sur l'état actuel des équations aux différences finies", Bull. des Sc. Math. 1920, p. I74 et suiv.

${ }^{2}$ N. Nielsen, Math. Ann. Bd. 59, pp. 103-106.

${ }^{3}$ C. Guichard, Ann. de l'École Normale, I887.

${ }^{4}$ P. A PPELL, Journal de Liouville, I89I.

${ }^{5}$ A. Hurwitz, Acta mathematica, t. 20. 
à la théorie, en remplaçant les polynomes de Bernoulli par des fonctions entières, convenablement choisies, qui assurent la convergence du développement de la solution, lorsque $g(x)$ est une fonction entière quelconque.

Mais c'est à $M$. Nörlund qu'on doit l'aspect moderne de la théorie, qui crée des formules sommatoires, tombées en oubli, un puissant instrument de recherche et qui en obtient la solution, qu'il nomme principale - en ne faisant que des hypothèses très générales sur la nature particulière de la fonction donnée $g(x)-$ de l'équation fonctionnelle (A).

Les travaux de M. Nörlund sur l'équation (A), sur l'équation

$$
f(x+\mathrm{I})+f(x)=2 g(x)
$$

et sur les équations obtenues en itérant les opérateurs des premiers membres ${ }^{6}$, sont fondamentaux pour la nouvelle théorie.

Les méthodes, si fécondes, de M. Nörlund ont permis l'extension de la théorie à des équations plus générales que celles de $M$. Nörlund et je dois citer, suivant le degré de généralité, le mémoire de M. R. Raclis ${ }^{7}$ et celui de M. S. Bochner $^{8}$ qui y étudie l'équation aux différences finies

$$
\underset{p}{\operatorname{E}} \boldsymbol{q}(x)=A_{0} \boldsymbol{\varphi}(x)+A_{1} \varphi\left(x+\omega_{1}\right)+\cdots+A_{p} \boldsymbol{g}\left(x+\omega_{p}\right)=g(x) .
$$

C'est cette même équation qui forme l'objet du présent travail. J'y introduis les polynomes $G_{n}^{k}(x)^{9}$, qui ont une grande parenté avec ceux de M. Bochner, mais dont je détermine la valeur approchée pour $n$ très grand

Je retrouve par des moyens un peu différents les résultats de M. Bochner et qui ont trait à la théorie de M. Nörlund.

J'étudie aussi quelques solutions spéciales, obtenues en étendant les procédés de $\mathrm{A}$. Hurwitz et à cet effet, j’introduis une suite de fonctions entières $\left(S_{n}^{k}(x)^{10}\right.$, nécessaires à l'étude.

Le problème des solutions périodiques, étudié pour l'équation (A) par $\mathbf{M}$. E. Picard, s'y trouve aussi étudié pour l'équation générale (C).

J'étudie aussi un cas spécial, celui où la résolution de l'équation fonction-

${ }^{6}$ N. NöRLund; Acta mathematica, I920, t. 43, pp. 121-196; Idem, 1922, t. 44, pp.7I-212; Trans. of the American Math. Society, v. 25, January 1923, pp. 13-98.

7 R. RACLIS, Acta mathematica, t. 55.

$8 \mathrm{~S}$. BOCHNER, Idem, t. $5 \mathrm{I}$.

"M. Ghermanesco, Bull. de l'Acad. de Relgique, 1933, 4, p. 387.

${ }^{10}$ Id., Rend. Accad. dei Lincei, 1933, mars, p. 38I. 
nelle $(\mathrm{C})$ se réduit à celle d'une infinité d'équations intégrales de première espèce, du type de M. Volterra.

Je montre encore comment on pourrait étudier une équation fonctionnelle, telle que (C), mais dans laquelle les $A_{i}$ sont des constantes asymptotiques.

Je finis, en considérant quelques systèmes d'équations fonctionnelles linéaires et en ramenant leur résolution à celle des équations, telles que (C).

\section{Chapitre I.}

\section{Les polynomes $G_{n}^{k}(x)$ et $\bar{G}_{n}^{k}(x)$.}

1. Considérons l'équation aux différences finies

$$
\underset{p}{\stackrel{x}{E}} \boldsymbol{F}=A_{0} F(x)+A_{1} F\left(x+\omega_{1}\right)+\cdots+A_{p} F\left(x+\omega_{p}\right)=g(x)
$$

dans laquelle les $A_{i}$ désignent des constantes, dont deux au moins sont différentes de zéro, les $\omega_{i}$ sont également des constantes, différentes ou non ${ }^{11}$, mais positives ou à parties réelles positives, cas auquel on parvient toujours par un simple changement de variable; désignons encore par $k$ le plus petit entier positif, tel qu'en posant

$$
B_{m}=A_{1} \omega_{1}^{m}+A_{2} \omega_{2}^{m}+\cdots+A_{p} \omega_{p}^{m}, \quad B_{0}=\sum_{0}^{p} A_{i}
$$

on ait $B_{k} \neq 0^{12}$ Quant au second membre $g(x)$, il désignera diverses fonctions que nous spécifierons dans la suite.

Pour l'étude de l'équation fonctionnelle (I), nous définissons les polynomes $G_{n}^{k}(x)$, solutions des équations particulières

$$
\begin{array}{lll}
\underset{p}{E} F=\frac{x^{n-k}}{(n-k) !} & \text { si } & n \geq k \\
\stackrel{x}{E} F=0 & \text { si } & n<k
\end{array}
$$

11 On peut bien les supposer toutes différentes, car dans le cas contraire on groupe les termes correspondants en un seul.

${ }^{12}$ Cette expression des $B_{m}$ rectifie légèrement celle considérée par M. Bochner (loc. eit. 8). 31-33617. Acta mathematica. 62. Imprimé le 20 december 1933. 
M. S. Bochner utilise (loc. cit. 8) pour l'étude de la même équation fonctionnelle (I) les polynomes $P_{n}(x)$ qu'il définit comme solutions des équations (4) et

$$
\underset{p}{\boldsymbol{E}} \boldsymbol{F}=x^{n-k}
$$

avec les mêmes conditions pour $n$. Il est facile d'établir un lien entre nos polynomes $G_{n}^{k}(x)$ et les polynomes $P_{n}(x)$ de M. Bochner: on voit que la différence

$$
P_{n}(x)-(n-k) ! G_{n}^{k}(x)
$$

est une solution de l'équation (4) et comme elle est un polynome, celui-ci ne peut être que du $(k-\mathrm{I})^{e}$ degré au plus.

Les raisons qui nous déterminent à employer les polynomes $G_{n}^{k}(x)$ au lieu des $P_{n}(x)$ sont bien simples: ils offrent plus de symétrie aux expressions qui les contiennent et, ce qui plus est, on peut leur trouver une valeur asymptotique, ce qui est fondamental dans l'étude de l'équation (I).

Remarquons en passant qu'on a toujours $k<p$ car autrement, le déterminant du système d'équations homogènes

$$
A_{1} \omega_{1}^{i}+A_{2} \omega_{\underline{1}}^{i}+\cdots+A_{p} \omega_{p}^{i}=0 \quad i=0, \mathrm{I}, \ldots p-\mathrm{I},
$$

qui n'est autre que le déterminant de Vandermonde des quantités $\omega_{i}$, serait nul, et il ne peut pas être nul sans que deux de ces constantes soient égales, hypothèse exclue (notice I I).

En revenant aux polynomes $G_{n}^{k}(x)$, on trouve en dérivant

$$
\frac{d G_{n}^{k}(x)}{d x}=G_{n-1}^{k}(x)
$$

ce qui montre que les polynomes $G_{n}^{k}(x)$ forment une suite $d^{\prime}$ Appell. ${ }^{13} \quad$ La formule de Taylor

devient, à cause de (6)

$$
G_{n}^{k}(x+h)=\sum_{0}^{\mathfrak{n}} \frac{h^{i}}{i !} \frac{d^{i} G_{n}^{k}(x)}{d x^{i}}
$$

$$
G_{n}^{k}(x+h)=\sum_{0}^{n} \frac{h^{i}}{i !} G_{n \rightarrow i}^{k}(x)
$$

${ }^{13}$ P. Appell, Ann. de l'École Norm. (1880), t. IV, pp. II9-I45. Voir aussi N. Nieises, Recherches sur les polynomes d'Hermite (Det Kgl. Danske V. S. 1918, I, 6). 
Définissons les nombres $G_{n}^{k}=G_{n}^{k}(0)$, cette relation nous donne

$$
G_{n}^{k}(x)=\sum_{0}^{n} G_{i}^{k} \frac{x^{n-i}}{(n-i)}
$$

Compte tenu de (8), l'équation fonctionnelle (3) devient

$$
\frac{B_{k}}{k !} G_{n-k}^{k}(x)+\frac{B_{k+1}}{(k+1) !} G_{n-k-1}^{k}(x)+\cdots+\frac{B_{n}}{n !} G_{0}^{k}(x)=\frac{x^{n-k}}{(n-k) !}
$$

ou, en changeant $n$ en $n+k$

$$
\frac{B_{k}}{k !} G_{n}^{k}(x)+\frac{B_{k+1}}{(k+\mathrm{I}) !} G_{n-1}^{k}(x)+\cdots+\frac{B_{n+k}}{(n+k) !} G_{0}^{k}(x)=\frac{x^{n}}{n !}
$$

qu'on peut considérer comme étant la relation de récurrence entre les polynomes $G_{n}^{k}(x)$ et qui permet leur détermination explicite, en procédant de proche en proche.

On a par exemple, pour $n=\mathrm{O}, \mathrm{I}$,

$$
\begin{aligned}
& G_{0}^{k}(x)=\frac{k !}{B_{k}} \\
& G_{1}^{k}(x)=\frac{k !}{B_{k}}\left(\frac{x}{\mathrm{I} !}-\frac{B_{k+1}}{(k+\mathrm{I}) \boldsymbol{B}_{k}}\right)
\end{aligned}
$$

La relation (I I) nous donne encore, pour $x=0$,

$$
\frac{\boldsymbol{B}_{k}}{k !} G_{n}^{k}+\frac{\boldsymbol{B}_{k+1}}{(k+\mathrm{I}) !} G_{n-1}^{k}+\cdots+\frac{\boldsymbol{B}_{n+k}}{(n+k) !} G_{0}^{k}=0 \quad n>0
$$

avec

$$
G_{0}^{k}=G_{0}^{n}(x)=\frac{k !}{B_{k}}
$$

et qui constitue la relation de récurrence entre les nombres $G_{i}^{k}$.

Si dans (I I) on remplace $n$ succesivement par $\mathrm{O}, \mathrm{I}, 2, \ldots n$, on obtient un système d'équations aux inconnues $G_{i}^{k}(x)$, duquel on tire l'expression suivante pour le polynome $G_{n}^{k}(x)$ 
(I 3)

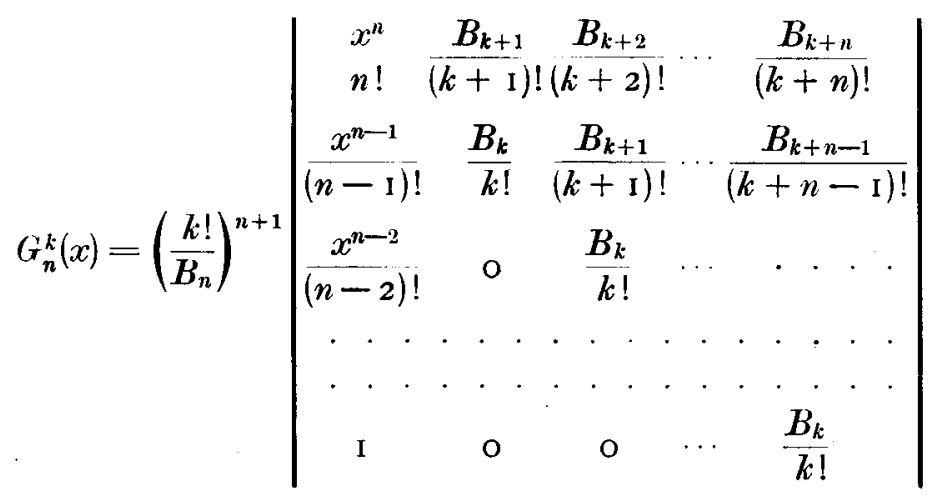

2. Si $k=0$, on peut trouver une expression remarquable pour les polynomes $G_{n}^{0}(x)=G_{n}(x)$. En effet, on déduit successivement de la relation (I I)

$$
\begin{aligned}
& G_{1}(x)=\frac{\mathrm{I}}{B_{0}}\left(x-\frac{B_{1}}{B_{0}}\right) \\
& G_{2}(x)=\frac{\mathrm{I}}{B_{0}}\left[x^{2}-\frac{\mathrm{I}}{B_{0}}\left(2 B_{1} x+B_{2}\right)+\frac{2 B_{1}^{2}}{B_{0}^{2}}\right] .
\end{aligned}
$$

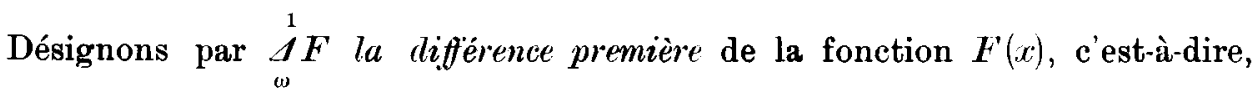
l'expression

$$
\stackrel{1}{\boldsymbol{H}} \boldsymbol{F}=\boldsymbol{F}(x+\omega)-F(x)
$$

et en général

$$
\underset{\omega_{1}\left(\omega_{2} \ldots \omega_{m}\right.}{\Delta}=\stackrel{1}{\mathcal{A}}\left(\begin{array}{c}
m-1 \\
\Delta F \\
\left(\omega_{1} \ldots \omega_{m}\right.
\end{array}\right)
$$

nous aurons

$$
\begin{aligned}
& 2 B_{1} x+B_{2}=\sum_{1}^{p} A_{i}\left(2 x \omega_{i}+\omega_{i}^{2}\right)=\sum_{1}^{p} A_{i} \dot{A}_{i}^{1} x^{2} \\
& 2 B_{1}^{2}=2\left(\sum_{1}^{p} A_{i} \omega_{i}\right)^{2}=\sum_{p} A_{i}^{z}\left(2 \omega_{i}^{*}\right)+2 \sum A_{i} A_{j}\left(2 \omega_{i}\left(\omega_{j}\right)\right.
\end{aligned}
$$

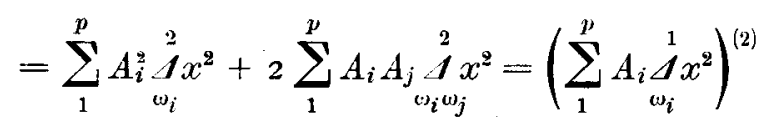

où l'on convient de remplacer les puissances symboliques 
Sur les équations aux différences finies.

245

on a done

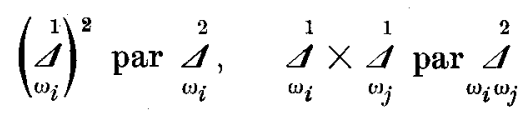

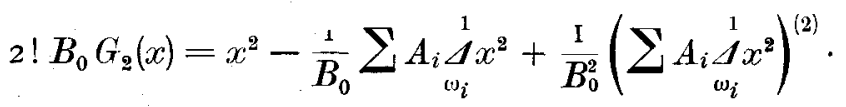

Je dis que, d'une manière générale, on a

(I4)

$$
\begin{aligned}
n ! B_{0} G_{n}(x)=x^{n}-\frac{\mathrm{I}}{B_{0}} \sum A_{i} A_{i} x^{n} & +\frac{\mathrm{I}}{B_{0}^{2}}\left(\sum A_{i} \Delta_{i} x^{n}\right)^{(2)}-\cdots \\
& +\frac{(-\mathrm{I})^{n}}{B_{0}^{n}}\left(\sum \underset{\omega_{i}}{\left.A_{i} \Delta x^{n}\right)^{(n)}}\right.
\end{aligned}
$$

En effet, supposons cette relation vraie pour les valeurs $\mathrm{I}, 2, \ldots n-\mathrm{I}$ de $n$; nous allons montrer qu'elle est encore vraie pour $n$. La relation (I I), qui pour $k=\mathrm{o}$ s'écrit

$\left(\mathrm{I} \mathbf{I}_{\mathbf{a}}\right)$

$$
B_{0} G_{n}(x)+\frac{B_{1}}{\mathrm{I} !} G_{n-1}(x)+\cdots+\frac{B_{n}}{n !} G_{0}(x)=\frac{x^{n}}{n !},
$$

devient, avec nos hypothèses

$\left(\mathrm{I} \mathrm{I}_{\mathrm{b}}\right)$

$$
\begin{aligned}
& n ! B_{0}^{2} G_{n}(x)=B_{0} x^{n}-\left(\begin{array}{c}
n \\
\mathrm{I}
\end{array}\right) B_{1}\left[x^{n-1}-\frac{\mathrm{I}}{B_{0}} \sum \underset{\left.{ }^{\prime}\right)_{i}}{A_{i} x^{n-1}}+\cdots\right] \\
& -\left(\begin{array}{l}
n \\
2
\end{array}\right) B_{2}\left[x^{n-2}-\frac{\mathrm{I}}{B_{0}} \sum \underset{\omega_{i}}{\left.A_{i} \mathcal{A} x^{n-2}+\cdots\right]}\right. \\
& -\left(\begin{array}{l}
n \\
n
\end{array}\right) B_{n} \\
& =B_{0} x^{n}-\left[\left(\begin{array}{l}
n \\
\mathrm{I}
\end{array}\right) B_{1} x^{n-1}+\left(\begin{array}{l}
n \\
2
\end{array}\right) B_{2} x^{n-2}+\cdots\left(\begin{array}{l}
n \\
n
\end{array}\right) B_{n}\right]
\end{aligned}
$$

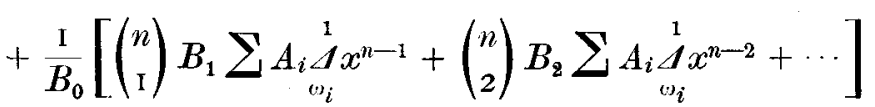

$$
\begin{aligned}
& -\frac{\mathrm{I}}{B_{0}^{2}}\left[\left(\begin{array}{c}
n \\
\mathrm{I}
\end{array}\right) B_{1}\left(\sum A_{i} \dot{\omega}_{i}^{1} x^{n-1}\right)^{(2)}+\cdots\right] \\
& +\frac{(-\mathrm{I})^{m-1}}{B_{0}^{m}}\left[\left(\begin{array}{l}
n \\
\mathrm{I}
\end{array}\right) B_{1}\left(\sum A_{i} \mathcal{A}_{\omega_{i}}^{1} x^{n-1}\right)^{(m)}+\cdots\right]+\cdots
\end{aligned}
$$


mais on a

$$
\begin{gathered}
\left(\begin{array}{l}
n \\
1
\end{array}\right) B_{1} x^{n-1}+\left(\begin{array}{l}
n \\
2
\end{array}\right) B_{2} x^{n-2}+\cdots+\left(\begin{array}{l}
n \\
n
\end{array}\right) B_{n}= \\
\sum A_{i}\left[\left(\begin{array}{l}
n \\
1
\end{array}\right) x^{n-1} \omega_{i}+\left(\begin{array}{l}
n \\
2
\end{array}\right) x^{n-2} \omega_{i}^{n}+\cdots\left(\begin{array}{l}
n \\
n
\end{array}\right) \omega_{i}^{n}\right]= \\
\sum A_{i}\left[\left(x+\omega_{i}\right)^{n}-x^{n}\right]=\sum A_{i} \dot{\Delta} x^{n} .
\end{gathered}
$$

Je dis qu'on a la relation générale

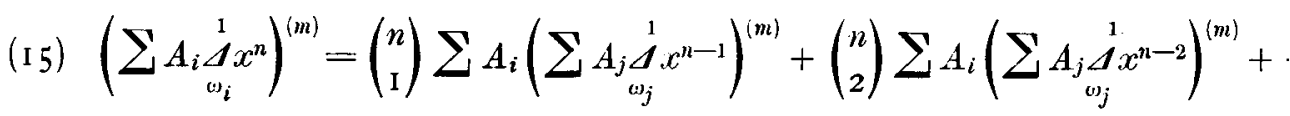

Nous pouvons la supposer vraie pour les entiers $1,2, \ldots m$; en lui appliquant l'opération $\sum A_{i} \dot{A}$ dans les deux membres, on aura visiblement

$$
\left(\sum A_{i \omega_{i}} \dot{L}^{n}\right)^{(m+1)}=\left(\begin{array}{c}
n \\
\mathrm{I}
\end{array}\right) \sum A_{i}\left(\sum A_{i} \dot{\omega}_{i} x^{n-1}\right)^{(m+1)}+\cdots
$$

et avec cela, ( $\left.\mathrm{I}_{\mathrm{b}}\right)$ n'est autre chose que (14).

Une telle relation a été donnée par M. R. Raclis pour ses polynomes particuliers $R_{m}^{p}(x)$ (loc. cit. $\tau$ ).

3. On trouve une expression semblable pour la solution de l'équation fonctionnelle (I) lorsque $g(x)$ est un polynome du $m^{e}$ degré, toujours dans l'hypothèse $k=0$. En écrivant

$$
g(x+h)=\sum_{0}^{m} \frac{h^{i}}{i !} g^{(i)}(x)=\sum_{0}^{m} g^{(i)}(x) \underset{p}{\stackrel{h}{E}} G_{i}(h)
$$

on voit aisément que la solution principale de l'équation (I) avec $g(x)$ polynome est le polynome

$$
\varphi(x+h)=\sum_{0}^{m} g^{(i)}(x) G_{i}(h)
$$

Nous n'avons qu'à remplacer dans cette relation, les polynomes $G_{i}(h)$ par leurs expressions (I4); on aura 


$$
\begin{aligned}
& B_{0} \varphi(x+h)=g(x)+\frac{g^{\prime}(x)}{\mathrm{I} !}\left[h-\frac{\mathrm{I}}{B_{0}} \sum A_{i} \stackrel{1}{\dot{A} h}\right] \\
& +\frac{g^{\prime \prime}(x)}{2 !}\left[h^{2}-\frac{\mathrm{I}}{\bar{B}_{0}} \sum \underset{{ }^{\prime}{ }_{i}}{A_{i}} h^{2}+\frac{\mathrm{I}}{B_{0}^{2}}\left(\sum \underset{\omega_{i}}{A_{i}\left\langle h^{2}\right.}\right)^{(2)}\right] \\
& =\left[g(x)+\frac{h}{1} g^{\prime}(x)+\frac{h^{2}}{2 !} g^{\prime \prime}(x)+\cdots\right]
\end{aligned}
$$

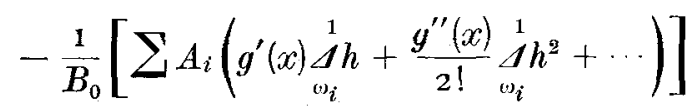

mais

$$
\begin{aligned}
& g(x)+\frac{h}{\mathrm{I}} g^{\prime}(x)+\frac{h^{2}}{2 !} g^{\prime \prime}(x)+\cdots=g(x+h) \\
& g^{\prime}(x) \underset{\omega_{i}}{\stackrel{1}{\Delta} h}+\frac{g^{\prime \prime}(x)}{2 !} \underset{\omega_{i}}{\Delta} h^{2}+\cdots={ }_{\omega_{i}}^{\Delta} g(x+h)
\end{aligned}
$$

et on démontre, par induction, la relation générale

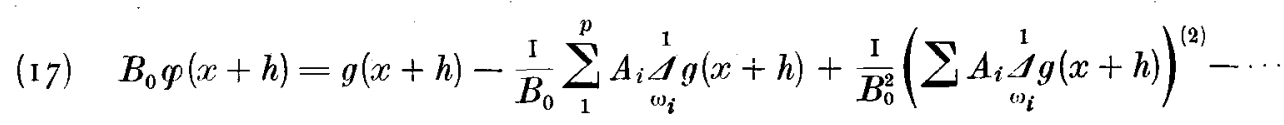

le développement s'arrétant de lui-même au $(m+\mathrm{I})^{\mathrm{e}}$ terme.

4. On peut déterminer une valeur approchée du polynome $G_{n}^{k}(x)$ pour $n$ très grand. Désignons par $\omega$ la plus grande des quantités $\omega_{i}$, on leur plus grand module si quelques unes sont complexes, par $A$ la somme des modules des coefficients $A_{i}$, de sorte qu'on aura

$$
\left|B_{i}\right|=\left|\sum_{1}^{p} A_{j} \omega_{j}^{i}\right|<\sum_{1}^{p}\left|A_{j}\right| \omega^{i}<A \omega^{i}
$$

La relation (I I) nous permet d'écrire pour les valeurs successives de $n$ et compte tenu de (I 8)

$$
\begin{aligned}
\left|B_{k} G_{0}^{k}(x)\right| & =k ! \\
\left|B_{k}^{2} G_{1}^{k}(x)\right| & <k !\left[\frac{x}{\mathrm{I} !}\left|B_{k}\right|+\frac{\left|B_{k+1}\right|}{(k+\mathrm{I}) !}\left|B_{k} G_{0}^{k}(x)\right|\right] \\
& <k !\left[A \omega^{k} \frac{x}{\mathrm{I} !}+\frac{A \omega^{k+1}}{(k+\mathrm{I}) !} k !\right] \Longrightarrow k ! A \omega^{k}\left[\frac{x}{\mathrm{I} !}+\frac{\omega}{k+\mathrm{I}}\right]
\end{aligned}
$$




$$
\begin{aligned}
\left|B_{k}^{3} G_{y}^{k}(x)\right| & <k !\left[\left|B_{k}^{z}\right| \frac{x^{2}}{2 !}+\frac{\left|B_{k+1}\right|}{(k+\mathrm{I}) !}\left|B_{k}^{z} G_{1}^{k}(x)\right|+\frac{\left|B_{k} B_{k+2}\right|}{(k+2) !}\left|B_{k} G_{0}^{k}(x)\right|\right] \\
& <k !\left[A^{2} \omega^{2 k} \frac{x^{2}}{2 !}+\frac{A^{2} \omega^{2 k+1}}{k+\mathrm{I}}\left(\frac{x}{\mathrm{I}}+\frac{\omega}{k+\mathrm{I}}\right)+\frac{A^{2} \omega^{2 k+2}}{(k+1)^{2}}\right] \\
& =k ! A^{2} \omega^{2 k}\left[\frac{x^{2}}{2 !}+\frac{\omega x}{k+\mathrm{I}}+\frac{2 \omega^{2}}{(k+\mathrm{I})^{2}}\right] .
\end{aligned}
$$

On trouve de même

$$
\left|B_{k}^{4} G_{s}^{k}(x)\right|<k ! A^{3} \omega^{3 k}\left[\frac{x^{3}}{3 !}+\frac{\omega x^{2}}{k+\mathrm{I}}+\frac{2 \omega^{2} x}{(k+\mathrm{I})^{2}}+\frac{4 \omega^{3}}{(k+\mathrm{I})^{3}}\right] .
$$

Je dis qu'on a, d'une manière générale

$$
\begin{gathered}
\left|B_{k}^{n+1} G_{n}^{k}(x)\right|< \\
<k ! A^{n} \omega^{n k}\left[\frac{x^{n}}{n !}+\frac{\omega x^{n-1}}{(k+\mathrm{I})(n-1) !}+\frac{2 \omega^{2} x^{n-2}}{(k+\mathrm{I})^{2}(n-2) !}+\cdots+\frac{2^{n-1}\left(\omega^{n}\right.}{(k+\mathrm{I})^{n}}\right] .
\end{gathered}
$$

Il suffit de supposer cette relation vraie pour les valeurs $0, \mathrm{I}, 2, \ldots n$ de $n$, pour démontrer qu'on obtient une limitation analogue pour le polynome $G_{n+1}^{k}(x)$.

De la relation (I I) on déduit pour le polynome $G_{n+1}^{k}(x)$,

$$
\begin{gathered}
\left|B_{k}^{n+2} G_{n+1}^{k}(x)\right| \\
<k !\left[\left|B_{k}^{n+1}\right| \frac{x^{n+1}}{(n+\mathrm{I}) !}+\frac{\left|B_{k+1}\right|}{(k+\mathrm{I}) !}\left|B_{k}^{n+1} G_{n}^{k}(x)\right|+\cdots+\frac{\left|B_{k}^{n} B_{n+k+1}\right|}{(n+k+\mathrm{I}) !}\left|B_{k} G_{o}^{k}(x)\right|\right] \\
<k ! A^{n+1} \omega^{k(n+1)}\left[\frac{x^{n+1}}{(n+\mathrm{I}) !}+\frac{\omega}{k+\mathrm{I}}\left(\frac{x^{n}}{n !}+\frac{\omega x^{n-1}}{(k+\mathrm{I})(n-\mathrm{I}) !}+\cdots+\frac{2^{n-1} \omega^{n}}{(k+\mathrm{I})^{n}}\right)\right. \\
\left.+\frac{\omega^{2}}{(k+\mathrm{I})^{2}}\left(\frac{x^{n-1}}{(n-\mathrm{I}) !}+\frac{\omega x^{n-2}}{(k+\mathrm{I})(n-2) !}+\cdots+\frac{2^{n-2} \omega^{n-1}}{(k+\mathrm{I})^{n-1}}\right)+\cdots\right] \\
<k ! A^{n+1} \omega^{k(n+1)}\left[\frac{x^{n+1}}{(n+1) !}+\frac{\omega x^{n}}{(k+1) n !}+\frac{2 \omega^{2} x^{n-1}}{(k+\mathrm{I})^{2}(n-\mathrm{I}) !}+\cdots+\frac{2^{n} \omega^{n+1}}{(k+\mathrm{I})^{n+1}}\right]
\end{gathered}
$$

expression de la même forme que (19), qui est ainsi générale. On a encore

$$
\begin{aligned}
\left|B_{k}^{n+1} G_{n}^{k}(x)\right| & <k ! A^{n} \omega^{k n}\left[\frac{x^{n}}{n !}+\frac{2 \omega x^{n-1}}{(k+\mathrm{I})(n-1) !}+\cdots+\frac{2^{n} \omega^{n}}{(k+\mathrm{I})^{n}}\right] \\
& =k !\left(\frac{2 A \omega^{k+1}}{k+\mathrm{I}}\right)^{n}\left[\mathrm{I}+\frac{(k+\mathrm{I}) x}{2 \omega \mathrm{I} !}+\frac{(k+\mathrm{I})^{2} x^{2}}{(2 \omega)^{2} 2 !}+\cdots+\frac{(k+\mathrm{I})^{n} x^{n}}{(2 \omega)^{n} n !}\right]
\end{aligned}
$$


En posant

$$
a=\frac{k !}{\left|B_{k}\right|}, \quad M=\frac{2 A \omega^{k+1}}{(k+\mathrm{I})\left|B_{k}\right|}, \lambda=\frac{k+\mathrm{I}}{2 \omega}
$$

la dernière inégalité devient

$$
\left|G_{n}^{k}(x)\right|<a M^{n} e^{\lambda x}
$$

et elle va jouer un rôle important dans la suite.

Si $x$ prend aussi des valeurs négatives on remplacera $x$ dans le second membre de (21) par $|x|$.

5. Définissons encore les polynomes $\bar{G}_{n}^{k}(x)$ par la relation

$$
\underset{p}{E}\left(x^{n}\right)=n ! \bar{G}_{n}^{k}(x), \quad \bar{G}_{n}^{k}(x)=0 \text { pour } n<k
$$

qui montre qu'on peut obtenir $\bar{G}_{n}^{k}(x)$ en appliquant au polynome $G_{n+k}^{k}(x)$ l'opération $\underset{p}{\stackrel{x}{E}}$ deux fois de suite.

De la relation (22) on déduit

$$
\begin{gathered}
n ! \bar{G}_{n}^{k}(x)=\left(\begin{array}{l}
n \\
k
\end{array}\right) B_{k} x^{n-k}+\left(\begin{array}{c}
n \\
k+\mathrm{I}
\end{array}\right) B_{k+1} x^{n-k-1}+\cdots+\left(\begin{array}{l}
n \\
k
\end{array}\right) B_{n} \\
n \geq k .
\end{gathered}
$$

Appliquons l'opération $\underset{p}{\stackrel{h}{E}}$ aux deux membres de la relation (8); on a

$$
(x+h)^{n-k}=(n-k) ! \sum_{i=k}^{n} \bar{G}_{i}^{k}(h) G_{n-i}^{k}(x)
$$

et comme, d'après (23), on a $n ! \bar{G}_{n}^{k}(0)=B_{n}$, nous obtenons pour $h=$ o la relation (10), pour $x=0$ la relation

$$
x^{n-k}=(n-k) ! \sum_{i=k}^{n} G_{n-i}^{k} \bar{G}_{i}^{k}(x)
$$

qui constitue la relation de récurrence entre les polynomes $\bar{G}_{n}^{k}(x)$ et pour $x=h=0$ la relation

$$
\sum_{i=k}^{n} \frac{B_{i}}{i !} G_{n-i}^{k}=\left\{\begin{array}{l}
\mathrm{o} \\
\mathrm{I}
\end{array}\right.
$$

32-33617. Acta mathematica. 62. Imprimé le 20 december 1933 . 
suivant que $n>k, n=k$ et qui d'ailleurs n'est autre que (I2). De la relation (25) on déduit l'expression suivante pour les polynomes $\widetilde{G}_{n}^{k}(x)$

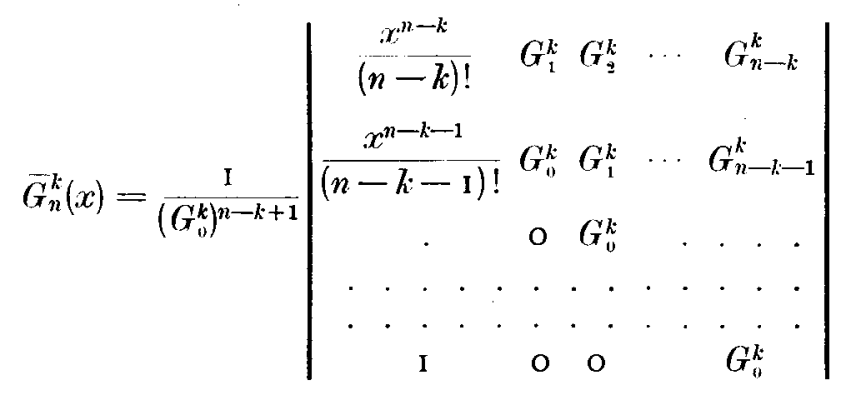

On a aussi

$$
\frac{d \bar{G}_{n}^{k}(x)}{d x}=\bar{G}_{n-1}^{k}(x)
$$

qui, avec (25), montre que les polynomes $\bar{G}_{n}^{k}(x)$ sont des solutions particulières des équations différentielles

$$
G_{n-k}^{k} \frac{d^{n-k} y}{d x^{n-k}}+G_{n-k-1}^{k} \frac{d^{n-l-1} y}{d x^{n-k-1}}+\cdots+G_{n}^{k} y=\frac{x^{n-k}}{(n-k) !} .
$$

On aurait trouvé de même, que les polynomes $G_{n}^{k}(x)$ sont des intégrales particulières des équations différentielles

$$
\frac{B_{n+k}}{(n+k) !} \frac{d^{n} y}{d x^{n}}+\underset{(n+k-1) !}{B_{n+k-1}} \frac{d^{n-1} y}{d x^{n-1}}+\cdots+\frac{B_{k}}{k !} y=\frac{x^{n}}{n !} .
$$

6. Nous pouvons déterminer une valeur approchée du polynome $\bar{G}_{n}^{k}(x)$ pour $n$ très grand. On peut écrire la relation (23), compte tenu de (18)

$$
\begin{aligned}
n !\left|\bar{G}_{n}^{k}(x)\right| & <\left(\begin{array}{l}
n \\
k
\end{array}\right)\left|B_{k}\right||x|^{n-k}+\left(\begin{array}{c}
n \\
k+\mathrm{I}
\end{array}\right)\left|B_{k+1}\right||x|^{n-k-1}+\cdots+\left|B_{n}\right| \\
& <A \omega_{k}\left[\left(\begin{array}{l}
n \\
k
\end{array}\right)|x|^{n-k}+\left(\begin{array}{c}
n \\
k+\mathrm{I}
\end{array}\right) \omega|x|^{n-k-1}+\cdots+\omega^{n-k}\right]
\end{aligned}
$$

mais on a

$$
\left(\begin{array}{c}
n \\
k+i
\end{array}\right)<n !\left(\begin{array}{c}
n-k \\
i
\end{array}\right) \quad \cdot \quad i \leq n-k
$$

donc 


$$
\left|\bar{G}_{n}^{k}(x)\right|<A \omega^{k}\left[|x|^{n-k}+\left(\begin{array}{c}
n-k \\
\mathbf{I}
\end{array}\right) \omega|x|^{n-k-1}+\cdots+\left(\begin{array}{c}
n-k \\
n-k
\end{array}\right) \omega^{n-k}\right]
$$

ou

$$
\left|\bar{G}_{n}^{k}(x)\right|<A \omega^{k}(|x|+\omega)^{n-k}
$$

\section{Formules sommatoires.}

7. Nous allons mettre en évidence quelques développements particuliers en série de polynomes $G_{n}^{k}(x)$.

Considérons le développement

$$
e^{t x}=\sum_{0}^{\infty} \frac{x^{i}}{i !} t^{i}
$$

et appliquons l'opération $\underset{p}{\stackrel{x}{E}}$ dans les deux membres de cette relation; en désignant par $O(t)$ l'expression

$$
O(t)=A_{0}+A_{1} e^{t \omega_{1}}+A_{2} e^{t \omega_{2}}+\cdots+A_{p} e^{t \omega_{p}}
$$

rencontrée aussi par M. Bochner dans ses recherches, on obtient.

$$
O(t) e^{t x}=\sum_{k}^{\infty} t^{i} \bar{G}_{i}^{k}(x)
$$

et le deuxième membre représente une série convergente si, d'après (3I), on a

$$
|t(|x|+\omega)|<\mathbf{I}
$$

On déduit de (33)

$$
O(t)=\sum_{k}^{\infty} \frac{B_{i}}{i !} t^{i}
$$

ce qu'on pouvait obtenir directement de (32).

Formons le produit

$$
\left(\sum_{0}^{\infty} G_{i}^{k} t^{i}\right) \times\left(\sum_{k}^{\infty} \frac{B_{i}}{i !} t^{i}\right)
$$

les coefficients des termes en $t^{\lambda}(\lambda>k)$ sont tous nuls, en vertu de la relation (26), celui de $t^{k}$ étant égal à $u n$; on obtient ainsi la relation 


$$
\frac{t^{k}}{O(t)}=\sum_{0}^{\infty} G_{i}^{k} t^{i}
$$

Multiplions les deux membres de cette relation par $e^{t k}$, on a

$$
\frac{t^{i} e^{i x}}{O(t)}=\left(\sum_{0}^{\infty} \frac{t^{i} x^{i}}{i !}\right) \times\left(\sum_{0}^{\infty} G_{i}^{k} t^{i}\right) .
$$

Dans le deuxième membre, le coefficient de $t^{i}$ n'est autre, d'après la relation (9), que le polynome $G_{i}^{k}(x)$, done

$$
\frac{t^{k} e^{t x}}{O(t)}=\sum_{0}^{x} t^{i} G_{i}^{k}(x)
$$

et l'inégalité (2I) montre que le deuxième membre converge si

$$
|t M|<\mathrm{I} .
$$

8. L'inégalité (2I) montre encore que la série

$$
G(x, t)=\sum_{0}^{\infty} \frac{t^{n}}{n !} G_{n}^{k}(x)
$$

converge pour toute valeur de $x$ et de $t$ et représente, par suite, une fonction entière. On ne peut pas déterminer l'expression finie de la fonction génératrice $G(x, t)$; elle est probablement ${ }^{-}$une transcendante étroitement liée à la fonction bien connue de Bessel, car on peut l'exprimer à l'aide de cette fonction. On a, en effet, d'après la relation (9),

$$
\begin{aligned}
G(x, t) & =\sum_{0}^{\infty} \frac{t^{n}}{n !}\left[G_{0}^{k} \frac{x^{n}}{n !}+G_{1}^{k} \frac{x^{n-1}}{(n-\mathrm{I}) !}+\cdots+G_{n}^{k}\right] \\
& =G_{0}^{k} \sum_{0}^{\infty} \frac{t^{n} x^{n}}{n ! n !}+G_{1}^{k} \sum_{1}^{\infty} \frac{t^{n} x^{n-1}}{n !(n-\mathrm{I}) !}+G_{2}^{k} \sum_{2}^{\infty} \frac{t^{n} x^{n-2}}{n !(n-2) !}+
\end{aligned}
$$

Mais, en considérant la fonction de Bessel $J(1, Z)$, on a

$$
J(\mathrm{I}, t x)=\mathrm{I}+\frac{t x}{1 ! \mathrm{I} !}+\frac{t^{2} x^{2}}{2 ! 2 !}+\cdots
$$

on en déduit 


$$
\begin{aligned}
& \sum_{0}^{\infty} \frac{t^{n} x^{n}}{n ! n !}=J(\mathrm{I}, t x) \\
& \sum_{1}^{\infty} \frac{t^{n} x^{n-1}}{n !(n-\mathrm{I}) !}=\frac{d}{d x} J(\mathrm{I}, t x) \\
& \sum_{2}^{\infty} \frac{t^{n} x^{n-2}}{n !(n-2) !}=\frac{d^{2}}{d x^{2}} J(\mathrm{I}, t x)
\end{aligned}
$$

de sorte qu'on a aussi

$$
G(x, t)=\sum_{0}^{\infty} G_{i}^{k} \frac{d^{i} J(\mathrm{1}, t x)}{d x^{i}}
$$

On a d'ailleurs

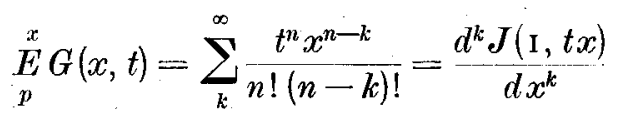

et aussi

$$
\underset{p}{E} G(x, t)=\sum_{k}^{\infty} G_{n}^{k}(x) \bar{G}_{n}^{k}(t)
$$

On peut encore regarder la transcendante entière $G(x, t)$ comme la solution d'une équation intégrale du type de M. Volterra

$$
G(x, t)+\lambda \int_{0}^{t} N(t-s) G(x, s) d s=\frac{k !}{B_{k}} J(\mathrm{I}, t x) ; \quad \lambda=\frac{k !}{\bar{B}_{k}}
$$

dans laquelle $x$ est considéré comme paramètre et

$$
N(u)=\frac{B_{k+1}}{(k+\mathrm{I}) !}+\frac{B_{k+2}}{\mathrm{I} !(k+2) !} u+\frac{B_{k+3}}{2 !(k+3) !} u^{2}+
$$

En effet, l'identité

$$
\int_{0}^{t}(t-s)^{\alpha} s^{\beta} d s=\frac{\alpha ! \beta ! t^{\alpha+\beta+1}}{(\alpha+\beta+\mathrm{I}) !}
$$

montre que le coefficient de $\frac{t^{n}}{n !}$ dans le premier membre de l'équation intégrale considérée eșt 


$$
\frac{k !}{\boldsymbol{B}_{k}}\left[\frac{B_{k}}{k !} G_{n}^{k}(x)+\frac{\boldsymbol{B}_{k+1}}{(k+1) !} G_{n-1}^{k}(x)+\cdots+\frac{B_{k+n}}{(k+n) !} G_{0}^{k}(x)\right]
$$

c'est-à-dire, d'après (I I), justement $\frac{k ! x^{n}}{n ! B_{k}}$ qui est le coefficient du même terme dans le second membre de (43).

La série qui représente le noyau $N(u)$ est entière, car on a, d'après (18)

$$
\begin{gathered}
|N(u)|<\frac{A \omega^{k+1}}{(k+1) !}\left[\mathrm{I}+\frac{\omega|u|}{\mathrm{I} !(k+2)}+\frac{\omega^{2}|u|^{2}}{2 !(k+2)^{2}}+\cdots\right] \\
|N(u)|<-\frac{A \omega^{k+1} e^{K|u|}}{(k+1) !}, \quad K=\frac{\omega}{k+2} .
\end{gathered}
$$

Comme la solution de l'équation intégrale (43) est dans ces conditions, d'après la théorie de $M$. Volterra, une fonction entière, on aurait déduit cette propriété de la fonction $G(x, t)$ sans connaitre l'inégalité (2I).

9. Nous avons considéré deux développements particuliers en série de polynomes $G_{n}^{k}(x)$ et étudié leur convergence. Mais si nous nous donnons une fonction arbitraire $\varphi(x)$, dans quelles conditions peut-elle être développée en série de ces polynomes?

La réponse est donnée par les formules sommatoires, qui, quoique connues du temps d'Euler, ne se sont revélées comme de puissants instruments de recherche que depuis les travaux de M. Nörlund.

M. Bochner a donné une formule sommatoire, attachée à l'équation fonctionnelle (I), à l'aide des polynomes $P_{n}(x)$; sa méthode s'inspire des procédés de M. Nörlund.

Je vais établir la formule sommatoire pour les polynomes $G_{n}^{k}(x)$ par une autre méthode, de laquelle on déduira facilement la formule de M. Bochner et toutes les autres, qui se rattachent à des équations fonctionnelles plus particulières que (I).

Nous allons imposer à la fonction donnée $\varphi(x)$ la condition d'avoir des dérivées jusqu'à un certain ordre $m+$ I. La formule de Taylor

(46) $\varphi(x+h)=\varphi(x)+\frac{h}{\mathrm{I}} \boldsymbol{\varphi}^{\prime}(x)+\cdots+\frac{h^{m}}{m !} \boldsymbol{\varphi}^{(m)}(x)+\int_{0}^{h} \frac{(h-s)^{m}}{m !} \boldsymbol{\varphi}^{(m+1)}(x+s) d s$

nous permet d'écrire 
Sur les équations aux différences finies.

255

(47) $\underset{p}{\stackrel{x}{E}} \varphi(x)=\frac{B_{k}}{k !} \varphi^{(k)}(x)+\frac{B_{k+1}}{(k+\mathrm{I}) !} \varphi^{(k+1)}(x)+\cdots$

$$
+\frac{B_{m}}{m !} \varphi^{(m)}(x)+\sum_{0}^{p} A_{i} \int_{0}^{\omega_{i}} \frac{\left(\omega_{i}-s\right)^{m}}{m !} g^{(m+1)}(x+s) d s .
$$

Posons $\varphi(x)=f^{(k)}(x)$ et remplaçons dans (46) les $\frac{h^{i}}{i !}$ par les expressions données par la relation (I I) dans laquelle on a fait $x=h$; on obtient, après avoir groupé convenablement

$$
\begin{aligned}
& \varphi(x+h)=G_{0}^{k}(h)\left[\frac{B_{k}}{k !} f^{(k)}(x)+\frac{B_{k+1}}{(k+\mathrm{I}) !} f^{(k+1)}(x)+\cdots+\frac{B^{k+m}}{(k+m) !} f^{(k+m)}(x)\right] \\
& +G_{1}^{k}(h)\left[\frac{B_{k}}{k !} f^{(k+1)}(x)+\frac{B_{k+1}}{(k+\mathrm{I}) !} f^{(k+2)}(x)+\cdots+\frac{B_{k+m-1}}{(k+m-\mathrm{I}) !} f^{(k+m)}(x)\right] \\
& \text {. . . . . . . . . . . . . . . . . . . } \\
& +G_{m+k}^{k}(h)\left[\frac{B_{k}}{k !} f^{(m+k)}(x)\right]+\int_{0}^{h} \frac{(h-s)^{m}}{m !} \varphi^{(m+1)}(x+s) d s
\end{aligned}
$$

ou encore, vu la relation (47), dans laquelle on remplace $m$ successivement par $m+k, m+k-\mathrm{I}, \ldots$

$$
\begin{aligned}
\varphi(x+h) & =\sum_{j=0}^{m+k} G_{j}^{k}(h) \underset{p}{E} f^{(j)}(x)+\int_{0}^{h} \frac{(h-s)^{m}}{m !} p^{(m+1)}(x+s) d s \\
& -\sum_{j=0}^{m+k} G_{j}^{k}(h) \sum_{i=0}^{p} A_{i} \int_{0}^{\omega_{i}} \frac{\left(\omega_{i}-s\right)^{m+k-j}}{(m+k-j) !} \varphi^{(m+1)}(x+s) d s .
\end{aligned}
$$

En désignant par $R_{k}$ la dernière double somme, on a

$$
R_{k}=\sum_{j=0}^{m+k} G_{j}^{k}(h) \sum_{i=0}^{p} A_{i} \int_{0}^{\omega_{i}} \frac{\left(\omega_{i}-s\right)^{m+k-j}}{(m+k-j) !} \varphi^{(m+1)}(x+s) d s=
$$


256

M. Ghermanesco.

$$
\begin{aligned}
& =\sum_{i=0}^{p} A_{i} \int_{0}^{\omega_{i}} \varphi^{(m+1)}(x+s)\left[\sum_{j=0}^{m+k} \frac{\left(\omega_{i}-s\right)^{m+k-j}}{(m+k-j) !} G_{j}^{k}(h)\right] d s= \\
& =\sum_{i=0}^{p} A_{i} \int_{0}^{\omega_{i}} G_{m+k}^{k}\left(h-s+\omega_{i}\right) \varphi^{(m+1)}(x+s) d s .
\end{aligned}
$$

Posons

(49) $R_{m+1}^{k}=\int_{0}^{h} \frac{(h-s)^{m}}{m !} \varphi^{(m+1)}(x+s) d s-\sum_{i=0}^{p} A_{i} \int_{0}^{(j)_{i}} G_{m+k}^{k}\left(h-s+\omega_{i}\right) \varphi^{(m+1)}(x+s) d s$

la formule sommatoire $(48)$ devient

$(50)$

$$
\varphi(x+h)=\sum_{j=0}^{m+k} G_{j}^{k}(h) \underset{p}{E} f^{(j)}(x)+R_{m+1}^{k}, f^{(k)}(x)=\varphi(x)
$$

qui, pour $k=0$, se réduit à la suivante

$(5 \mathrm{I})$

$$
\varphi(x+h)=\sum_{j=0}^{m} G_{j}(h) \underset{p}{\stackrel{x}{E}} \varphi^{(j)}(x)+R_{m+1}
$$

On peut donner d'autres formes au terme reste $R_{m+1}^{k}$. D'après (I I) on peut écrire

$$
\begin{aligned}
R_{m+1}^{k} & =\sum_{i=0}^{p} A_{i} \int_{0}^{h} G_{m+k}^{k}\left(h-s+\omega_{i}\right) \varphi^{(m+1)}(x+s) d s- \\
& -\sum_{i=0}^{p} A_{i} \int_{0}^{\omega_{i}} G_{m+k}^{k}\left(h-s+\omega_{i}\right) \varphi^{(m+1)}(x+s) d s \\
= & \sum_{i=0}^{p} A_{i}\left[\int_{0}^{h} G_{m+k}^{k}\left(h-s+\omega_{i}\right) \varphi^{(m+1)}(x+s) d s-\right. \\
& \left.-\int_{0}^{\omega_{i}} G_{m+k}^{k}\left(h-s+\omega_{i}\right) \varphi^{(m+1)}(x+s) d s\right]
\end{aligned}
$$




$$
R_{m+1}^{k}=\sum_{i=0}^{p} A_{i} \int_{\omega_{i}}^{h} G_{m+k}^{k}\left(h-s+\omega_{i}\right) \varphi^{(m+1)}(x+s) d s .
$$

10. Supposons maintenant que $\omega_{i}, h, x$ soient réels et $o \leq h<\omega, \omega$ étant la plus grande des constantes $\omega_{i}$; lorsque $s$ varie de $h$ à $\omega_{i}, h-s+\omega_{i}$ varie de $\omega_{i}$ à $h$. Désignons par $\dot{G}_{m}^{k}(x)$ la fonction définie pour toute valeur réelle de $x$ par l'équation

$$
\underset{p}{\stackrel{x}{E}} \dot{G}_{n}^{k}(x)=0
$$

et qui dans l'intervalle $\mathrm{o} \leq x<\omega$ coïncide avec le polynome $G_{n}^{k}(x)$; on peut alors remplacer dans $\left(5_{2}\right)$ le polynome $G_{n}^{k}\left(h-s+\omega_{i}\right)$ par la fonction $\dot{G}_{n}^{k}\left(h-s+\omega_{i}\right)$

$$
R_{m+1}^{k}=\sum_{i=0}^{p} A_{i} \int_{\omega_{i}}^{h} \dot{G}_{m+k}^{k}\left(h-s+\omega_{i}\right) \varphi^{(m+1)}(x+s) d s .
$$

Décomposons l'intégrale en $\int_{0}^{h}+\int_{\omega_{i}}^{0}$; on aura

$$
\begin{aligned}
R_{m+1}^{k} & =\int_{0}^{h} \sum_{i=0}^{p} A_{i} \dot{G}_{m+n}^{k}\left(h-s+\omega_{i}\right) \varphi^{(m+1)}(x+s) d s \\
& +\sum_{i=0}^{p} A_{i} \int_{\omega_{i}}^{0} \dot{G}_{m+k}^{k}\left(h-s+\omega_{i}\right) \varphi^{(m+1)}(x+s) d s
\end{aligned}
$$

La première intégrale est nulle, d'après (53); dans ce qui reste, décomposons encore l'intégrale en $\int_{\infty}^{0}+\int_{\omega_{i}}^{\infty} ;$ on aura

$$
\begin{aligned}
R_{m+1}^{k} & =\int_{\infty}^{0} \sum_{i=0}^{p} A_{i} \dot{G}_{m+n}^{k}\left(h-s+\omega_{i}\right) \varphi^{(m+1)}(x+s) d s \\
& +\sum_{i=0}^{p} A_{i} \int_{\omega_{i}}^{\infty} \dot{G}_{m+k}^{k}\left(h-s+\omega_{i}\right) \varphi^{(m+1)}(x+s) d s .
\end{aligned}
$$

33-33617. Acta mathematica. 62. Inprimé le 17 janvier 1934. 
La première intégrale est encore nulle, d'après (53); en faisant dans l'autre le changement de variable $s=\omega_{i}+t$, on obtient comme expression du terme reste

$$
R_{m+1}^{k}=\int_{0}^{\infty} \dot{G}_{m+k}^{k}(h-t) \sum_{i=0}^{p} \varphi^{(m+1)}\left(x+t+\omega_{i}\right) d t
$$

ou encore

$$
R_{m+1}^{k}=\int_{0}^{\infty} \dot{G}_{m+k}^{k}(h-t) \underset{p}{\stackrel{x}{L}} \varphi^{(m+1)}(x+t) d t
$$

L'étude de la convergence de cette intégrale a été faite par M. Bochner à l'aide de la valeur asymptotique de la fonction $\overline{\boldsymbol{P}}_{n}(x) \sim c_{n} \dot{G}_{n}^{k}(x)$.

Nous en verrons plus loin les conditions nécessaires.

$$
\text { III. L'équation } \underset{p}{\underset{p}{E} \boldsymbol{F}}=g(x) \text {. }
$$

11. L'équation fonctionnelle ( $\mathrm{r}$ )

$$
\underset{p}{\stackrel{x}{E}} \boldsymbol{H}=g(x)
$$

a été résolue, suivant le procédé de $\mathbf{M}$. Nörlund, par M. Bochner, à l'aide des formules sommatoires. Nous nous proposons d'examiner ici d'autres modes de solution, obtenus en étendant quelques-uns de ceux connus pour les équations particulières

$$
F(x+1) \pm F(x)=g(x) .
$$

Remarquons d'abord que si les écarts $\omega_{i}$ ont des rapports mutuels commensurables, l'équation (I) peut être écrite

$$
A_{0} F(x)+A_{1} F(x+\omega)+A_{2} F(x+2 \omega)+\cdots+A_{p} F(x+p \omega)=g(x)
$$

et une telle équation est ramenée à une suite d'équations de la forme élémentaire

$$
\begin{gathered}
\varphi_{i}(x+\omega)-\lambda_{i+1} \varphi_{i}(x)=\varphi_{i+1}(x) \\
i=0,1,2, \ldots p-\mathrm{I}
\end{gathered}
$$

avec $\varphi_{p}(x)=g(x)$, les $\lambda_{i}$ étant les racines de l'équation caractéristique 


$$
S(\lambda)=A_{0} \lambda^{p}+A_{1} \lambda^{p-1}+\cdots+A_{p}=0 .
$$

La résolution successive des équations (55) nous conduit à la solution de l'équation $\left(\mathrm{I}^{\prime \prime}\right)$.

Mais nous allons écarter ce cas particulier, en nous maintenant dans l'hypothèse la plus générale en ce qui concerne les $\omega_{i}$.

Nous commencerons par chercher une solution formelle de l'équation fonctionnelle (I), quelle que soit la fonction donnée $g(x)$. Posons $A_{0} G(x)=g(x)$ et introduisons un paramètre $\lambda$; l'équation (I) s'écrit

$$
\varphi(x)-\lambda\left[A_{1} \varphi\left(x+\omega_{1}\right)+A_{2} \varphi\left(x+\omega_{2}\right)+\cdots+A_{p} \varphi\left(x+\omega_{p}\right)\right]=G(x) .
$$

Cherchons à y satisfaire par un développement de la forme

$$
\varphi(x)=\varphi_{0}(x)+\lambda \varphi_{1}(x)+\lambda^{2} \varphi_{2}(x)+\cdots
$$

nous obtenons les relations

$$
\begin{aligned}
& \varphi_{0}(x)=G(x) \\
& \varphi_{1}(x)=A_{1} G\left(x+\omega_{1}\right)+A_{2} G\left(x+\omega_{2}\right)+\cdots+A_{p} G\left(x+\omega_{p}\right) \\
& \varphi_{2}(x)=A_{1} \varphi_{1}\left(x+\omega_{1}\right)+A_{2} \varphi_{1}\left(x+\omega_{2}\right)+\cdots+A_{p} \varphi_{1}\left(x+\omega_{p}\right) \\
& (\varphi) \ldots \ldots \ldots \ldots \text {. . . . . . . . . } \\
& \varphi_{n}(x)=A_{1} \varphi_{n-1}\left(x+\omega_{1}\right)+A_{2} \varphi_{n-1}\left(x+\omega_{2}\right)+\cdots+A_{p} \varphi_{n-1}\left(x+\omega_{p}\right)
\end{aligned}
$$

Nous pouvons mettre les fonctions $\varphi_{u}(x)$ sous une forme assez simple: on a

$$
\begin{aligned}
\varphi_{1}(x) & =\underset{p}{\stackrel{x}{E}} G(x)-A_{0} G(x)=\left(\underset{p}{\underset{E}{E}-A_{0}}\right) G(x) \\
\varphi_{2}(x) & =\underset{p}{\underset{x}{E}} \varphi_{1}(x)-A_{0} \varphi_{1}(x)=\underset{p}{\underset{x}{E}}\left[\underset{p}{E} G(x)-A_{0} G(x)\right]-A_{0} \underset{p}{E} G(x)+A_{0}^{2} G(x) \\
& =\frac{x}{E^{2}} G(x)-2 A_{0} \underset{p}{E} G(x)+A_{0}^{2} G(x)
\end{aligned}
$$

ou encore

$$
\varphi_{2}(x)=\left(\underset{p}{E}-A_{0}\right)^{(2)} G(x)
$$

Or, on aura en général 


$$
\varphi_{n}(x)=\left(\underset{p}{E}-A_{0}\right)^{(n)} G(x)
$$

En effet, cette relation étant supposée vraie, on a

$$
\varphi_{n}(x)=\underset{p}{\stackrel{x}{E^{n}}} G(x)-\left(\begin{array}{l}
n \\
1
\end{array}\right) A_{0} \underset{p}{\stackrel{x}{E^{n-1}}} G(x)+\left(\begin{array}{l}
n \\
2
\end{array}\right) \underset{p}{A_{0}^{2} \stackrel{x}{E}^{n-2}\left(\dot{x}(x)-\cdots+(-1)^{n}\right.}\left(\begin{array}{l}
n \\
n
\end{array}\right) A_{0}^{n} G(x) .
$$

En appliquant aux deux membres lopération $\left(\underset{p}{E}-A_{0}\right)$ on aura facilement

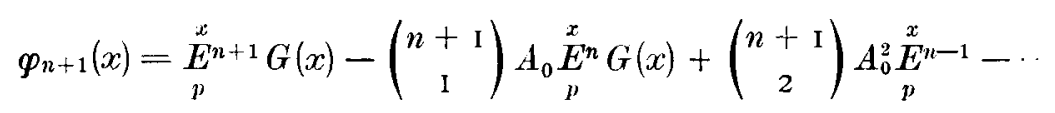

ou encore

$$
\varphi_{n+1}(x)=\left(\underset{p}{E}-A_{0}\right)^{(n+1)} G(x)
$$

ce qui prouve la généralité de (59). Le développement formel de la solution de l'équation fonctionnelle (57) sera par conséquent

$$
A_{0} \varphi(x)=\sum_{0}^{\infty} \lambda^{n}\left(\underset{p}{E}-A_{0}\right)^{(n)} g(x)
$$

et il n'y a qu'à y faire $A_{0} \lambda=-1$ pour obtenir celui de la solution de (I).

Supposons, pour en faire une application, $g(x)=e^{t x}$ et posons $\mu(t)=O(t)-A_{0}$; on aura

$$
\left(\underset{p}{E}-A_{0}\right)^{(n)} e^{t x}=e^{t x} \mu^{n}(t)
$$

done

$$
A_{0} \varphi(x)=e^{t x}\left[\mathrm{I}+\lambda \mu(t)+\lambda^{2} \mu^{2}(t)+\cdots\right]
$$

qui converge si

qui devient, pour $A_{0} \lambda=-\mathrm{I}$

$$
|\lambda \mu(t)|<\mathrm{I}
$$

$$
\left|\frac{O(t)}{A_{0}}\right|<2
$$

et qui conduit à la solution, déjà trouvée, de l'équation (I)

$$
\varphi(x)=\frac{e^{t x}}{O(t)} .
$$


Supposons encore que l'équation fonctionnelle (I) se réduise à la suivante

$$
\varphi(x+\omega) \pm \varphi(x)=g(x) .
$$

L'expression (60) de la solution formelle devient ici

$$
A_{0} \varphi(x)=\sum_{0}^{\infty}\left(\frac{-\mathrm{I}}{A_{0}}\right)^{n} g(x+n \omega)
$$

dans laquelle $A_{0}= \pm$ I et on retrouve ainsi les séries

$$
-\sum_{0}^{\infty} g(x+n \omega), \quad \sum_{0}^{\infty}(-\mathrm{I})^{n} g(x+n \omega)
$$

étudiées par M. Nörlund.

12. Dans le cas général de l'équation fonctionnelle ( $\mathrm{I}$ ) on peut encore mettre la solution formelle sous une autre forme: remplaçons, dans $(\varphi)$, l'expression de $\varphi_{1}(x)$ dans $\varphi_{2}(x)$, ensuite celle de $\varphi_{2}(x)$, ainsi obtenue, dans $\varphi_{3}(x)$ et ainsi de suite; on parvient à l'expression suivante pour l'approximation générale $\varphi_{n}(x)$

$$
\varphi_{n}(x)=\sum A_{1}^{n_{i 1}} A_{.2}^{n_{i 2}} \ldots A_{p}^{n_{i p}} g\left(x+\Omega_{n i}\right)
$$

avec

$$
\begin{gathered}
\Omega_{n i}=n_{i 1} \omega_{1}+n_{i 2} \omega_{2}+\cdots+n_{i p} \omega_{p} \\
i=\mathrm{I}, 2, \ldots p
\end{gathered}
$$

les $n_{i j}$ étant des entiers positifs, non tous nuls et tels que

$$
n_{i 1}+n_{i 2}+\cdots+n_{i p}=n .
$$

En posant encore

$$
A_{1}^{n_{i 1}} A_{2}^{n_{i 2}} \ldots A_{p}^{n_{i p}}=A_{n i},
$$

on obtient la solution formelle de l'équation (57) sous la forme

$$
A_{0} \varphi(x)=\sum_{n=0}^{\infty} \lambda^{n}\left[\sum_{i=1}^{p} A_{n i} g\left(x+\Omega_{n i}\right)\right]
$$


Une expression de cette forme, mais avec moins de précision en ce qui concernè les coefficients, a été donnée par M. Bochner.

13. Il s'agit maintenant d'étudier les cas de convergence de l'une des deux séries (6o) et (66), qui représentent la solution formelle de l'équation fonetionnelle (57).

Comme ces séries en sont uniques, elles devront conduire, en cas de convergence, à la solution principale, telle qu'elle a été définie par M. Nörlund et trouvée par M. Bochner pour l'équation fonctionnelle (57).

Or cette solution principale est aisée à retrouver, en partant de la formule sommatoire (50) avec l'expression (54) pour le terme reste $R_{m+1}^{k}$; écrivons l'équation fonctionnelle (I), dans laquelle on a remplacé la fonction $g(x)$ par son développement (50); on a

$$
\begin{gathered}
\underset{p}{\boldsymbol{x}} \boldsymbol{F}(x+h)=\sum_{j=0}^{n+k} G_{j}^{k}(h) \underset{p}{\stackrel{x}{E}} f^{(j)}(x)+\int_{0}^{x} \dot{G}_{m+k}^{k}(h-t) \underset{p}{\stackrel{x}{\boldsymbol{E}}} \boldsymbol{\phi}^{(m+1)}(x+t) d t \\
f^{(k)}(x)=g(x)
\end{gathered}
$$

ou encore

$$
\underset{p}{x} F(x+h)=\underset{p}{x}\left[\sum_{j=0}^{m+k} G_{j}^{k}(h) f^{(j)}(x)+\int_{0}^{x} \dot{G}_{m+k}^{k}(h-t) \varphi^{(m+1)}(x+t) d t\right]
$$

d'où il s'ensuit

$$
F(x+h)=\sum_{j=0}^{m+k} G_{j}^{k}(h) f^{(j)}(x)+\int_{0}^{x} \dot{G}_{m+k}^{k}(h-t) g^{(m+1)}(x+t) d t
$$

parce que les deux membres ne diffèrent que par une fonction arbitraire, qui est solution de l'équation

$$
\stackrel{x}{E} \boldsymbol{F}=\mathrm{o}
$$

et qui est identiquemment nulle, puisque $F(x+h)$ est la solution principale.

De sorte que, si les séries (6o) et (66) convergent pour $A_{0} \lambda=-\mathrm{I}$, leur somme commune doit se réduire au deuxième membre de (67).

Remarquons d'abord qu'on a

$$
\underset{p}{\stackrel{x}{E}} F=\left(\stackrel{x}{E}-A_{0}\right) F+A_{0} F
$$




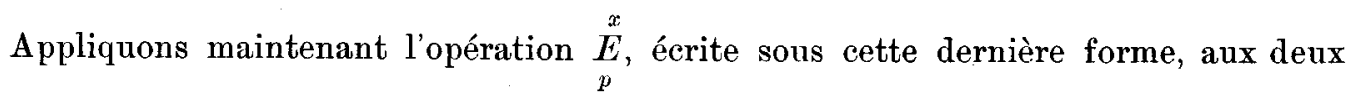
membres de la relation (6o); on aura

$$
A_{0} \underset{p}{\stackrel{x}{E}} \varphi(x)=\sum_{0}^{\infty} \lambda^{n}\left(\underset{p}{E}-A_{0}\right)^{(n+1)} g(x)+A_{0} \sum_{0}^{\infty} \lambda^{n}\left(\underset{p}{E}-A_{0}\right)^{(n)} g(x)
$$

ou, en remplaçant $\lambda$ par $-\frac{\mathrm{I}}{A_{0}}$

$$
A_{0} \underset{p}{\stackrel{x}{E}} \varphi(x)=A_{0} g(x)+\lim _{n=\infty} \lambda^{n}\left(\underset{p}{\mathbb{E}}-A_{0}\right)^{(n+1)} g(x)
$$

Cela établi, appliquons à la fonction $\varphi(x)$, donnée par (60), la formule sommatoire (50) avec l'expression (54) du terme reste $R_{m+1}^{k}$; compte tenu de la relation (68), on aura

$$
\begin{aligned}
A_{0} \varphi(x+h) & =\sum_{j=0}^{m+k} G_{j}^{k}(h)\left[A_{0} f^{(j)}(x)+\lim _{n=\infty} \lambda^{n}\left(\underset{p}{E}-A_{0}\right)^{(n+1)} f^{(j)}(x)\right] \\
& +\int_{0}^{\infty} \dot{G}_{m+k}^{k}(h-t)\left[A_{0} g^{(m+1)}(x+t)+\lim _{n=\infty} \lambda^{n}\left(\underset{p}{E}-A_{0}\right)^{(n+1)} g^{(m+1)}(x+t)\right] d t
\end{aligned}
$$

et il est facile de voir que, pour que cette relation conduise à la solution principale $(67)$, il faut et il suffit que l'on ait

$$
\begin{gathered}
\lim _{n=\infty} \lambda^{n}\left(\underset{p}{E}-A_{0}\right)^{(n)} f^{(j)}(x)=\mathrm{o} \\
j=\mathrm{O}, \mathrm{I}, 2, \ldots k+m+\mathrm{I} .
\end{gathered}
$$

Cette relation a lieu, d'après (62), lorsque

$$
\lim _{n=\infty} \lambda^{n} A_{n i} f^{(j)}\left(x+\Omega_{n i}\right)=0 .
$$

Or, soient $A, \omega$ les plus grandes valeurs absolues des constantes $A_{i}, \omega_{i}$; on a

$$
\left|A_{n i}\right|<A^{n}, \quad \Omega_{n i}<\omega n .
$$

La relation (7o) a lieu en même temps que la suivante

$$
\lim _{n=\infty}(\lambda A)^{n} f^{(j)}(x+n \omega)=0
$$


posons $x+n \omega=y$, cette relation devient

$$
(\lambda A)^{-\frac{x}{\omega}} \lim _{y=x}(\lambda A)^{\frac{y}{\omega}} f^{(j)}(y)=0
$$

Il suffit done d'avoir

$$
\lim _{x=x} x^{\alpha}(\lambda A)^{\frac{x}{\omega}} f^{(j)}(x)=0
$$

ce qui a lieu lorsque

$$
\lim _{x=x} \frac{f^{(j)}(x)}{x^{n}}=\mathrm{o}, \quad \begin{aligned}
& j=\mathrm{o}, \mathrm{I}, 2, \ldots m+k+\mathrm{I} \\
& n=\text { arbitrairement grand }
\end{aligned}
$$

pour que les relations (72), (7I), (70) et, par conséquent, (69) aient lieu, ce qui nous conduit de la solution formelle (6o) à la solution principale (67) de l'équation fonctionnelle (57).

La relation (73) contient aussi la condition pour que l'intégrale (54) ait un sens.

On peut aussi arriver à la solution principale en la définissant comme la limite pour $\eta=0$ de la série

$$
\sum_{0}^{\infty} \lambda^{n}\left[\sum_{i=1}^{p} A_{n i} e^{-\eta\left(x+\Omega_{n i}\right.} g\left(x+\Omega_{n i}\right)\right]
$$

$\eta$ tendant vers zéro par des valeurs décroissantes, ce qui revient à considérer la solution principale de l'équation fonctionnelle (I) comme la limite de la solution principale de la suivante

$$
\underset{p}{\stackrel{x}{E} \boldsymbol{F}}=e^{-\eta x} g(x) \quad \eta>0
$$

lorsque $\eta$ tend vers zéro. On arrive à un tel résultat en suivant de près la marche indiquée par $M$. Nörlund.

14. Un critère de convergence pour la série (66), dans le cas des variables complexes étudié pour le cas particulier des séries (6I) par M. R. D. Carmichael ${ }^{14}$, peut être mis en évidence en étendant le procédé de ce géomètre. Supposons que la fonction donnée $g(x)$ puisse se mettre sous la forme

14 R. D. Carmichael, American Journal, 1916, I9I7. Voir aussi Ta-Li, Journal für reine u. ang. Math. I933, H. 4, p. 87 . 


$$
g(x)=x^{P(x)} e^{Q(x)}\left(\mathrm{I}+\frac{a_{1}}{x}+\frac{a_{2}}{x^{2}}+\cdots\right)
$$

dans laquelle $P(x), Q(x)$ désignent des polynomes de la variable complexe $x$, de degrés respectivement $p$ et $q(p+q \geq 2)$ et soit $x_{0}$ un point pour lequel la série

$$
F(x)=\sum_{0}^{\infty} c_{n i} g\left(x+\Omega_{n i}\right)
$$

converge. Posons

$$
\nu_{n i}(x)=\frac{g\left(x+\Omega_{n i}\right)}{g\left(x_{0}+\Omega_{n i}\right)}
$$

On peut écrire

$$
g(x) \sim e^{P(x) \log x+Q(x)} \psi(x) \quad \lim _{x=x} \psi(x)=\mathbf{I}
$$

en posant aussi

$$
\begin{aligned}
& P(x)=\mu_{0}+\mu_{1} x+\cdots+\mu_{p} x^{p} \\
& Q(x)=v_{0}+\nu_{1} x+\cdots+\nu_{q} x^{q}
\end{aligned}
$$

et compte tenu du développement

$$
\log \left(\mathrm{I}+\frac{x}{\Omega_{n i}}\right)=\frac{x}{\Omega_{n i}}-\frac{\mathrm{I}}{2}\left(\frac{x}{\Omega_{n i}}\right)^{2}+\cdots
$$

dont nous prendrons le premier terme seulement, on pourra écrire encore

$$
g\left(x+\Omega_{n i}\right) \sim e^{A \log \Omega_{n i}+B} P_{1}\left(\frac{\mathrm{I}}{n}\right), \quad \lim _{n=\infty} P_{1}\left(\frac{\mathrm{I}}{n}\right)=\mathrm{I}
$$

avec

$$
\begin{aligned}
& A=\mu_{p} \Omega_{n i}^{p}+p \mu_{p-1} x \Omega_{n i}^{p-1}+\cdots \\
& B=\mu_{p} x \Omega_{n i}^{p-1}+\cdots+\boldsymbol{v}_{q} q x \Omega_{n i}^{q-1}+\cdots
\end{aligned}
$$

done

$$
\left|v_{n i}(x)\right|=\left|\frac{g\left(x+\Omega_{n i}\right.}{g\left(x_{0}+\Omega_{n i}\right.}\right| \sim\left|e^{p \mu_{p}\left(x-x_{0}\right) \Omega_{n i}^{p-1} \log \Omega_{n i}+\cdots+v_{q} q\left(x-x_{0}\right) \Omega_{n i}^{q-1}} P_{\mathbf{2}}\left(\frac{\mathrm{I}}{n}\right)\right|
$$

avec

$$
\lim _{n \rightarrow \infty} P_{2}\left(\frac{\mathrm{I}}{n}\right)=\mathrm{I}
$$

ou encore

34-33617. Aeta mathematica. 62. Imprimé le 19 janvier 1934. 


$$
\left|\Omega_{n i}^{2} \nu_{n i}(x)\right| \sim\left|e^{p \mu_{p}\left(x-x_{0}\right) \Omega_{n i}^{p-1} \log \Omega_{n i}+2 \log \Omega_{n i}+\cdots+v_{q} q\left(x-x_{0}\right) \Omega_{n i}^{q-1}} P_{2}\left(\frac{\mathrm{I}}{n}\right)\right|
$$

mais $\Omega_{n i} \sim n \omega^{:}$, donc

$$
\left|\Omega_{n i}^{2} \nu_{n i}(x)\right| \sim\left|e^{\left.p u_{p}\left(x-x_{0}\right)(0) n\right)^{p-1} \log n(1)+2 \log n(\omega)+\cdots+r_{q} q\left(x-x_{0}\right)\left(n(\omega)^{\prime / g}-1\right.} P_{2}\left(\frac{\mathrm{I}}{n}\right)\right|
$$

Si $p \geq q$, la partie principale de l'exposant du second membre est $p \mu_{p}\left(x-x_{0}\right) n^{p-1} \log n$. Pour que le second membre tende vers zéro, pour $n$ infini, il suffit donc que la partie réelle de l'expression $\mu_{p}\left(x-x_{0}\right)$ soit négative, c'est-à-dire

$$
\mathfrak{R}\left(\mu_{p} x\right)<\mathfrak{R}\left(\mu_{p} x_{0}\right)
$$

Cela étant, la série dont le terme général est $v_{n i}(x)$ est comparable à la suivante

$$
\sum \frac{\mathrm{I}}{\Omega_{n i}^{2}}
$$

qui est convergente puisque, en désignant par $\omega^{\prime}$ la plus petite des quantités positives $\omega_{i}$, on a

$$
\Omega_{n i}>\omega^{\prime}\left(n_{i 1}+n_{i 2}+\cdots+n_{i p}\right)=n \omega^{\prime}
$$

done

$$
\frac{\mathrm{I}}{\Omega_{n i}^{2}}<\frac{\mathrm{I}}{n^{2}\left(t^{\prime 2}\right.}
$$

dont le sécond membre est le terme général d'une série convergente.

Si $p<q$, la partie principale de l'exposant sera $q v_{q}\left(x-x_{0}\right) \Omega_{n i}^{q-1}$ et on est conduit à la même conclusion.

Si $p=q=$ I il faut $\mathfrak{R}\left[\mu_{1}\left(x-x_{0}\right)\right]<-2$.

Or, la série $\Sigma v_{n i}(x)$ étant convergente, il s'ensuit aussi la convergence de la série

$$
\Sigma\left|\nu_{n i}(x)-\nu_{n+1, i}(x)\right|
$$

et, d'après Dedekind, la convergence de cette série entraine celle de

$$
\sum_{0}^{\infty} e_{n i} g\left(x+\Omega_{n i}\right)
$$

\footnotetext{
* $\omega$ désigne ici une valeur moyenne entre la plus grande et plus petite des $\omega i$.
} 
On peut done énoncer la propriété suivante:

Soit, dans le champ de la variable complexe $x$, une fonction $g(x)$, admettant dans le domaine du point à l'infini le développement asymptotique suivant

$$
g(x)=x^{P^{2}(x)} e^{Q(x)}\left(\mathrm{I}+\frac{a_{1}}{x}+\frac{a_{2}}{x^{2}}+\cdots\right)
$$

$P(x), Q(x)$ désignant des polynomes, à coefficients complexes de degrés respectivement $p$ et $q(p+q \geq 2)$.

Soient $\mu_{p}, v_{q}$ les coefficients des termes du plus haut degré dans ces deux polynomes; en les désignant indistinctement par $\sigma$, la série

$$
\Sigma c_{n i} g\left(x+\Omega_{n i}\right)
$$

sera convergente si, $x_{0}$ étant un point de convergence, on a $\Re(\sigma x)<\Re\left(\sigma x_{0}\right)$, ou $\Re(\sigma x)-\Re\left(\sigma x_{0}\right)<-2$ si $p=q=\mathrm{I}$.

15. La solution principale, telle quelle a été conçue par M. Nörlund, a l'avantage d'être très générale, puisqu'elle n'exige aucune hypothèse concernant la nature de la fonction donnée, $g(x)$. Mais si l'on tient compte de la nature de cette fonction, on peut obtenir une solution de l'équation fonctionnelle ( $\mathrm{I}$ ) spécialément attachée à la nature de $g(x)$, en suivant des procédés employés par maints auteurs antérieurement à M. Nörlund. Les solutions, fournies par ces procédés, ne jouissent pas de la généralité et en même temps des propriétés caractéristiques de la solution principale de M. Nörlund; elles sont toutefois dignes d'intérêt, autant par elles-mêmes que par les procédés qui les découvrent, d'autant plus qu'on peut en extraire toujours la solution principale, lorsqu'elle est convergente.

Nous allons examiner de ce point de vue les cas où la fonction donnée $g(x)$ est entière ou méromorphe.

16. Supposons la fonction $g(x)$ entière, admettant un développement de la forme

$$
g(x)=\sum_{0}^{\infty} \frac{a_{n}}{n !} x^{n}
$$

L'équation fonctionnelle (I) s'écrit

$$
\underset{p}{\operatorname{Ex}} \boldsymbol{F}^{\prime}(x)=\sum_{0}^{\infty} a_{n} \underset{p}{x} G_{n+k}^{k}(x)
$$


d'où la solution principale formelle

$$
F(x)=\sum_{0}^{\infty} a_{n} G_{n+k}^{k}(x) .
$$

Etudions la convergence de la série du second membre. D’après l'inégalité (2 I) on peut écrire

$$
\sum_{0}^{\infty}\left|a_{n} G_{n+k}^{k}(x)\right|<a e^{\lambda . x} \sum_{n}^{\infty}\left|a_{n}\right| \lambda K^{n}
$$

La série du deuxième membre converge si

$$
\lim _{n=\infty}\left|\frac{a_{n+1}}{a_{n}}\right|<\frac{1}{M}
$$

ce qui entraine aussi la convergence uniforme de la série (78). Comme (78) est une série uniformément convergente de polynomes, elle définit une fonction entière $F(x)$, qui est la solution principale de l'équation fonctionnelle (1).

Pour donner un exemple, prenons le cas, déjà rencontré, $g(x)=e^{t x}$. L'équation (I) admet dans ce cas la solution, fonction entière

$$
F^{\prime}(x)=\sum_{0}^{\infty} t^{n} G_{n+k}^{k}(x)
$$

Si l'on avait pris $g(x)=t^{k} e^{t x}$, la solution serait

$$
r(x)=\sum_{0}^{\infty} t^{n} G_{n}^{k}(x)
$$

Ces deux séries convergent si, d'après (79), on a

$$
|t M|<\mathrm{I} .
$$

Un cas particulier de la condition (79) est

$$
\lim _{n=\infty} \sqrt[n]{a_{n}}=0 .
$$

Tel est par exemple le cas pour la fonction de Bessel

$$
g(x)=J(\gamma, x)=\sum_{0}^{\infty} n ! \gamma(\gamma+1) \cdots(\gamma+n-1)
$$


la solution correspondante de l'équation (1) est

$$
\boldsymbol{F}(x)=\sum_{0}^{\infty} \frac{G_{n+k}^{k}(x)}{\gamma(\gamma+\mathrm{I}) \cdots(\gamma+n-\mathrm{I})} .
$$

Nous pouvons donc énoncer la propriété suivante:

Si $g(x)$ est une fonction entière, admettant le développement

$$
g(x)=\sum_{0}^{\infty} a_{n} \frac{x^{n}}{n !}
$$

et $s i$

$$
\lim _{n=\infty}\left|\frac{a_{n+1}}{a_{n}}\right|<\frac{\mathrm{I}}{M}
$$

l'équation fonctionnelle

$$
\underset{p}{\stackrel{x}{E} F}=g(x)
$$

admet comme solution principale la fonction entière

$$
F(x)=\sum_{0}^{\infty} a_{n} G_{n+k}^{k}(x)
$$

1\%. Mais la condition (79) n'est pas remplie pour toutes les fonctions entières, de sorte que la série (78), qui représente la solution de l'équation fonctionnelle (I) n'est pas toujours uniformément convergente.

Il faut alors remplaçer les polynomes $G_{n}^{k}(x)$ par d'autres fonctions entières, qui assurent l'uniforme convergence de la série $(78)$, lorsque $g(x)$ est une fonction entière quelconque.

Ce procédé a été indiqué pour la première fois par C. Guichard ${ }^{3}$, et appliqué ensuite par P. Appell ${ }^{4}$ et surtout par A. Hurwitz ${ }^{5}$, dont nous étendrons l'idée à l'équation fonctionnelle (I).

A cet effet, remarquons que la relation (37)

$$
\frac{t^{k} e^{t x}}{O(t)}=\sum_{0}^{\infty} t^{i} G_{i}^{k}(x)
$$

\footnotetext{
Note 3

4 Note 4

${ }^{5}$ Note 5 .
} 
permet d'écrire, à l'aide de l'intégrale de Cauchy

$$
G_{n}^{k}(x)=\stackrel{\mathrm{I}}{2 \pi i} \int \frac{e^{z x}}{O(z)} \cdot \frac{d z}{z^{n-k+1}}
$$

l'intégrale étant étendue à un contour renfermant à son intérieur le pôle $z=0$ seulement. Mais la fonction $O(z)$ admet comme zéros d'ordre $k$ les racines de l'équation (lorsque les $\omega_{i}$ sont entiers; autrement $k^{\prime}=0$ )

e'est-à-dire

$$
e^{z}-\mathrm{I}=\mathrm{o}
$$

$$
z=2 k^{\prime} \pi i \quad\left(k^{\prime}=0, \pm \mathrm{I}, \pm 2, \ldots \pm n, \ldots\right)
$$

puisque $B_{0}=B_{1}=B_{z}=\cdots=B_{k-1}=0$, ainsi que ceux provenant de l'annulation des facteurs de $O(z)$, tels que

$$
e^{z}+\alpha_{v}, e^{z}-\beta_{r}, \quad \alpha_{r}, \beta_{r}>0
$$

et qui sont compris dans l'une des deux formules

$$
z=L \epsilon_{v} \pm\left(2 k^{\prime}+1\right) \pi i, z=L \beta_{v} \pm 2 k^{\prime} \pi i
$$

En suivant l'idée de A. Hurwitz, nous allons considérer la fonction entière $\mathfrak{B}_{n}^{k}(x)$, définie à l'aide de l'intégrale de Cauchy

$$
G_{n}^{k}(x)=\frac{1}{2 ; i} i \int \frac{e^{z x}}{O(z)} \frac{d z}{z^{n-k+1}}
$$

l'intégrale étant prise de long d'un contour enveloppant cette fois les points

$$
\begin{gathered}
z=2 k^{\prime} \pi i, \quad z=L \alpha_{v} \pm\left(2 k^{\prime}+\mathrm{I}\right) \pi i, z=L \beta_{v} \pm 2 k^{\prime} \pi i \\
k^{\prime}=0, \pm \mathrm{I}, \pm 2, \ldots \pm n
\end{gathered}
$$

l'intégrale sera alors égale à la somme des résidus, correspondant à chacun de ces pôles.

Il est facile de montrer que la fonction $\left(\mathfrak{G}_{n}^{k}(x)\right.$, ainsi définie, est une solution de l'équation fonctionnelle (3). En effet, en appliquant l'opérateur $\underset{p}{E}$ aux deux membres de (82), on trouve

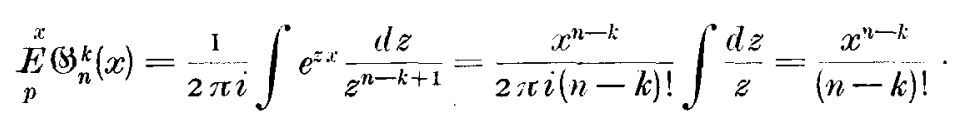

Il s'agit maintenant de déterminer une limite supérieure pour l'intégrale (82). 
Soit $a$ la plus grande valeur absolue des quantités $L \alpha_{v}, L \beta_{v}$; nous prendrons comme contour particulier $\dot{e}_{n}$ un carré, dont les côtés ont pour équations

$$
\xi= \pm a \pm(2 n+2) \pi, \quad \eta= \pm a \pm(2 n+2) \pi
$$

dans le plan de la variable complexe

$$
z=\xi+i \eta
$$

tel qu'il contienne à son intérieur tous les pôles envisagés plus haut et que nous ramenerons au carré fixe $\Gamma$, dont les côtés sont

$$
\xi^{\prime}= \pm \mathrm{I}, \quad \eta^{\prime}= \pm \mathrm{I}
$$

par le changement

$$
z-a(\mathrm{I}+i)=(2 n+2) \pi z^{\prime}, \quad z^{\prime}=\xi^{\prime}+i \eta^{\prime}
$$

on a sur $\Gamma$

$$
\left|a(\mathrm{I}+i)+(2 n+2) \pi z^{\prime}\right|>(2 n+2) \pi\left|z^{\prime}\right| \geq(2 n+2) \pi
$$

et si $x$ reste dans une région $R$, finie mais arbitrairement grande,

$$
e^{\left[a x(1+i)+(2 n+2) \pi x z^{\prime}\right]}<e^{a x}\left(e^{\pi x z^{\prime}}\right)^{2 n+2}<e^{a x} \varrho^{2 n+2}
$$

de sorte que

(84) $\quad\left|\mathfrak{S}_{n}^{k}(x)\right|<\frac{e^{a x} \varrho^{2 n+2}}{(2 n+2)^{n-k} \pi \pi^{n-k}}\left|\frac{\mathrm{I}}{2 \pi i} \int_{\Gamma} \frac{d z^{\prime}}{A_{0}+\sum_{1}^{p} A_{j} e^{\omega j} j\left[a(1+i)+(2 n+2) \pi z^{\prime}\right]}\right|$.

Pour évaluer la dernière intégrale, remarquons que le dénominateur, qui n'est autre que $O(z)$, peut être écrit

$$
O(z)=\left(e^{z}-\mathrm{I}\right)^{k}\left(e^{z}+\alpha_{v}\right)\left(e^{z}-\beta_{1}\right) \quad \nu=\mathrm{I}, 2, \ldots
$$

$z$ devant y être remplacé par son expression (83).

Or, on a

$$
\left|e^{a(i+1)+(2 n+2) \pi z^{\prime}}-\mathrm{I}\right|>\left|e^{a(i+1)+(2 n+2) \pi\left(\xi^{\prime}+i \eta^{\prime}\right)}\right|-\mathrm{I}>\left|e^{(2 n+2) \pi\left(\xi^{\prime}+i \eta^{\prime}\right)}\right|-\mathrm{I} .
$$

En évaluant le module de cette expression sur chacun des côtés du carré $\Gamma$, on 
trouve - tout aussi comme dans le cas particulier étudié par Hurwitz - qu'il est supérieur à $\frac{\mathrm{I}}{2}$.

On montre de la même manière que tous les autres facteurs de $O(z)$ sont supérieurs à des quantités fixes, ce qui conduit à la conclusion que $O(z)$ est anssi supérieure à une quantité fixe et par suite, l'intégrale qui figure dans l'inégalité $(84)$ est inférieure à une quantité $A$, ne dépendant pas de $n$, de sorte que (84) devient

$$
\left|\oiint_{n}^{k}(x)\right|<\frac{A e^{a x} \varrho^{2 n+2}}{(2 n+2)^{n-k} \pi^{n-k}}
$$

inégalité fondamentale pour la suite.

18. Reprenons l'équation fonctionnelle (I), dans laquelle $g(x)$ a le développement (77). Il est facile d'en déduire qu'une solution de l'équation (I) est donnée par

$$
F(x)=\sum_{0}^{\infty} a_{n} \mathfrak{G}_{n+k}^{k}(x)
$$

Etudions la convergence de la série du second membre. On peut écrire, d'après l'inégalité (85)

$$
\sum_{0}^{\infty}\left|a_{n}\right| \mid\left(5_{n+k}^{k}(x)\left|<\underline{Q}^{2 k+2} A e^{a x} \sum_{0}^{\infty}\right| a_{n} \mid \frac{\varrho^{2 n}}{(2 n+2)^{n} x^{n}} .\right.
$$

Pour décider de la convergence de la dernière série, nous allons chercher la limite de

$$
\sqrt[n]{\left|a_{n}\right|} \frac{e^{2}}{2 \pi(n+\mathrm{I})}
$$

pour $n$ infini. Or il est évident que le terme

$$
\frac{\varrho^{2}}{2 \pi(n+\mathrm{I})}
$$

tend vers zéro; il suffit que l'on ait

$$
\lim _{n=\infty} I^{n}\left|a_{n}\right|=\text { const. }
$$


pour que la série considérée et, par conséquent la série (86), converge uniformément. Comme (86) est une série de fonctions entières et comme elle converge uniformément pour toute valeur de $x$, sa somme $F(x)$ sera une fonction entière en $x$.

La condition (87) et le développement (77) montrent que $g(x)$ doit être une fonction entière, de sorte que nous pouvons énoncer la proposition suivante

Etaint donnée l'équation fonctionnelle (I) et les fonctions entières $g(x),\left(\mathcal{B}_{11}^{k}(x)\right.$, la fonction $F(x)$, donnée par le développement

$$
F(x)=\sum_{0}^{\infty} a_{n} \mathscr{G}_{n+k}^{k}(x)
$$

est une solution, fonction entière, de l'équation fonctionnelle (I).

19. La solution, ainsi trouvée, de l'équation fonctionnelle (I) n'est pas la solution principale; cependant, on peut en extraire la solution principale, lorsque la dernière est uniformément convergente. En effèt, séparons dans l'intégrale (82), qui définit la fonction $\mathfrak{G}_{n}^{k}(x)$, la partie correspondant au pôle $z=0$, des autres: la première est justement le polynome $G_{n}^{k}(x)$; quant à la partie restante, elle définit une fonction $g_{n}^{k}(x)$, qui est solution de l'équation fonctionnelle (4),

$$
\stackrel{x}{\underset{p}{E}} g_{n}^{k}(x)=\text { 。 }
$$

de sorte qu'on peut écrire

$$
\left(_{n}^{k}(x)=G_{n}^{k}(x)+g_{n}^{k}(x)\right.
$$

et la solution (86) de l'équation fonctionnelle (I) devient

$$
\boldsymbol{F}(x)=\sum_{0}^{\infty} a_{n} G_{n}^{k}(x)+\sum_{0}^{\infty} a_{n} g_{n}^{k}(x)
$$

et on reconnait, dans la première série du second membre, justement la solution principale (78), exprimée à l'aide des polynomes $G_{n}^{k}(x)$. Bien entendu, elle n'est pas uniformément convergente si la condition (79) n'est pas remplie, de sorte qu'on doit se contenter dans ce cas de la solution spéciale (86), exprimée à l'aide des fonctions entières $\mathscr{G}_{n}^{k}(x)$, si l'on ne veut pas avoir recours à la solution principale $(67)$.

35-33617. Acta mathematica. 62. Imprimé le 17 janvier 1934. 
20. On a mis ainsi en évidence une suite discrète de fonctions $g_{n}^{k}(x)$, solutions de l'équation fonctionnelle (4).

Pour en donner un exemple, considérons la fonction génératrice des polynomes d'Euler-Nörlund ${ }^{15}$

$$
\left(e^{-t w_{1}}+1\right)\left(e^{-t \omega_{2}}+1\right) \cdots\left(e^{n}+t w_{n}+\mathrm{I}\right)=\sum_{0}^{x} \frac{(-t)^{v}}{v !} E_{v}^{n}(x) .
$$

Cette fonction admet les pôles simples ${ }^{16}$

$$
t_{k^{\prime} j}=-\begin{array}{cl}
(2 k+\mathrm{I}) \pi i \\
\omega_{j}
\end{array} \quad \begin{aligned}
k^{\prime} & =0, \pm \mathrm{I}, \pm 2, \ldots \pm n, \ldots \\
j & =\mathrm{I}, 2, \ldots n
\end{aligned}
$$

avec les résidus

$$
\begin{gathered}
2^{n} e^{-t_{k^{\prime} j}{ }^{x}} \\
O_{1}^{\prime}\left(t_{k^{\prime} j}\right) \\
o_{1}(t)=\left(e^{-t \omega_{1}}+\mathrm{I}\right)\left(e^{-t \omega_{n}}+1\right) \cdots\left(e^{-t \omega_{n}} n+\mathrm{I}\right)
\end{gathered}
$$

done

$$
\mathfrak{G}_{v}^{n}(x)=E_{v}^{n}(x)+n ! 2^{n} \sum_{j=0}^{n} \sum_{k^{\prime}=0}^{ \pm n} \frac{e^{t_{k^{\prime}} j^{x}}}{O_{1}^{\prime}\left(t_{k^{\prime} j}\right)}
$$

21. Nous allons examiner maintenant le cas où la fonction $g(x)$ est méromorphe dans tout le plan, en étendant la méthode employée par Hurwitz* dans le cas particulier de l'équation fonctionnelle ( $\mathrm{I}^{\prime}$ ).

Remarquons d'abord qu'on peut écrire

$$
g(x)=g_{1}(x)+g_{2}(x)
$$

$g_{1}(x)$ n'ayant de pôles qu'à droite de l'axe $O \eta$ et $g_{2}(x)$ n'en ayant qu'à gauche de ce même axe. D'après cette remarque il suffira donc de déterminer les solutions des équations fonctionnelles

$$
\begin{aligned}
& \underset{p}{\stackrel{x}{E}} F_{1}=g_{1}(x) \\
& \underset{p}{\underset{E}{E}} F_{2}=g_{2}(x)
\end{aligned}
$$

${ }^{15}$ Loe. cit. 6 (troisième mémoire).

16 On suppose que les $\omega_{i}$ n'ont pas des rapports commensurables.

* Loc. cit. 5 . 
puisque la solution de l'équation ( $\mathrm{I}$ ) s'obtiendra en ajoutant ces deux solutions. On a, en effet, en posant $F(x)=F_{1}(x)+F_{2}(x)$,

$$
\underset{p}{\underset{E}{E}} F_{1}(x)+\underset{p}{\stackrel{x}{E}} F_{2}(x)=\underset{p}{\stackrel{x}{E}} F(x)=g_{1}(x)+g_{2}(x)=g(x) .
$$

Nous allons résoudre ainsi l'équation fonctionnelle (I) dans l'hypothèse que $g(x)$ n'ait de pôles, par exemple, qu'à gauche de l'axe $O \eta$. Dans ces conditions, la fonction $g\left(x+\Omega_{n i}\right)$ n'aura de pôles qu'à gauche de la droite

$$
\xi=-\Omega_{n i}
$$

Elle est alors holomorphe dans tout domaine borné, situé à droite de $\xi=-\Omega_{n i} ; \varepsilon_{n i}$ et $\delta$ étant deux nombres arbitrairement petits, on peut déterminer un polynome $g_{n i}(x)$, tel que

$$
\left|g_{n i}(x)-g\left(x+\Omega_{n i}\right)\right|<\varepsilon_{n i}
$$

dans toute région du demi-plan pour lequel on a

$$
|x|<\Omega_{n}-\delta,
$$

$\Omega_{n}$ étant le plus petit des $\Omega_{n i}$.

Soit alors la série

$$
F(x)=\sum_{n=0}^{\infty}\left(\frac{-\mathrm{I}}{A_{0}}\right)^{n} \sum_{i=1}^{p} A_{n i}\left[g_{n i}(x)-g\left(x+\Omega_{n i}\right)\right]
$$

et supposons qu'on ait chọisi les $\varepsilon_{n i}$ de manière que la série

$$
\sum_{0}^{\infty}\left(\frac{-\mathrm{I}}{A_{0}}\right)^{n} \sum_{1}^{p} A_{n i} \varepsilon_{n i}
$$

soit convergente. Soit $R$ une région bornée du plan, ne s'étendant pas, à gauche, au delà de la droite

$$
\xi=-N+\delta
$$

on aura dans cette région

$$
\left|g_{n i}(x)-g\left(x+\Omega_{n i}\right)\right|<\varepsilon_{n i}
$$

pourvu que

$$
\Omega_{n i}>N
$$


autrement dit, à partir du $N^{\mathrm{e}}$ terme, la série $F(x)$ converge uniformément; quant à la somme des $N^{+}$- I premiers termes, elle représente une fonction méromorphe. La région $R$ pouvant d'ailleurs être prise arbitrairement grande, pourvu qu'elle soit bornée, on voit que la série $\boldsymbol{F}(x)$ représente une fonction méromorphe dans tout le plan.

Si maintenant on tient compte de la relation (66), on peut écrire, en posant

$$
\begin{gathered}
\sum \sum\left(\begin{array}{c}
-\mathrm{I} \\
A_{0}
\end{array}\right)^{n} A_{n i} \underset{p}{\stackrel{x}{E} g_{n i}(x)=G(x),} \\
\underset{p}{E} \underset{p}{E} F(x)=g(x)+G(x) .
\end{gathered}
$$

Or, la condition (74) étant remplie par la fonction méromorphe $g(x)$, la sérue

$$
\sum \sum\left(\frac{-\mathrm{I}}{A_{0}}\right)^{n} A_{n i} g\left(x+Q_{n i}\right)
$$

est uniformément convergente dans la région définie plus haut; comme il y en a de même pour la fonction $F(x)$, il s'ensuit la même conclusion, d'après (89), pour

$$
F_{1}(x)=\sum \sum\left(\frac{-\mathrm{I}}{A_{0}}\right)^{n} A_{n i} g_{n i}(x)
$$

qui, étant une série de polynomes, définit par suite une fonction entière. On en déduit que la fonction $G(x)$ est aussi une fonction entière, puisque $G(x)=\underset{p}{E} F_{1}(x)$.

Cela établi, il suffit, d'après (90), de retrancher de la fonction $\boldsymbol{F}(x)$, donnée par (89), la fonction entière $\boldsymbol{F}_{1}(x)$, pour obtenir une solution, méromorphe dans tout le plan, de l'équation fonctionnelle ( $\mathrm{r}$ ).

On traite de la même manière le cas des pôles situés à droite de l'axe $O \eta$ et on détermine ainsi une solution de l'équation fonctionnelle ( $\mathrm{I}$ ) dans l'hypothèse faite au début sur la fonction $g(x)$.

22. Etudions maintenant le cas où l'équation fonctionnelle ( $\mathrm{I}$ ) admet des solutions périodiques. Ce problème a été traité pour la première fois par M. E. Picard pour l'équation fonctionnelle particulière ${ }^{17}$

$$
F(z+\omega)-\mu F(z)=P(z)
$$

17 E. PICARD, Acta mathematica, I 894 , t. I 8. 
Sa méthode s'étend, dans ses lignes générales, aussi à l'équation fonctionnelle générale (I).

Supposons la fonction $g(x)$ entière, $x$ étant complexe, admettant la période $i \omega$ et proposons-nous de déterminer une solution de l'équation fonctionnelle (I) admettant aussi la période $i \omega$.

Nous pouvons supposer, sans nuire à la généralité, $\omega$ réelle et positive.

Posons $X=e^{\frac{2 \pi x}{\omega}}$; la fonction entière $g(x)$, de période $i \omega$, peut se développer en série de Laurent ${ }^{18}$

$$
g(x)=\sum_{v=0}^{\infty}\left[a_{v} X^{v}+\frac{b_{v}}{X^{v}}\right]
$$

dans toute couronne de centre $O$.

Comme la solution cherchée $F(x)$ de l'équation (1) doit être entière, puisque $g(x)$ l'est, on aura aussi

$$
F(x)=\sum_{v=0}^{\infty}\left[c_{v} X^{v}+\frac{d_{v}}{X^{v}}\right]
$$

Remarquons qu'on a

$$
X(x+\alpha)=X(x) e^{\frac{2 \pi \alpha}{\omega}}
$$

de sorte qu'en posant

$$
2 \pi=t \omega
$$

on aura

$$
F\left(x+\omega_{i}\right)=\sum_{\nu=0}^{\infty}\left[c_{v} e^{v t \omega_{i}} X^{\nu}+\frac{d_{v}}{e^{v t} \frac{\omega_{i} X^{v}}{v}}\right]
$$

et l'équation fonctionnelle (I) devient

$$
\sum_{\nu=0}^{\infty}\left[c_{\nu} O(v t) X^{v}+\frac{d_{\nu} O(-v t)}{X^{\nu}}\right]=\sum_{v=0}^{\infty}\left[a_{v} X^{\nu}+\frac{b_{v}}{X^{\nu}}\right]
$$

Egalons les coefficients des mêmes puissances de $X$; on en obtient

$$
c_{v}=\frac{a_{v}}{O(\nu t)}, \quad d_{v}=\frac{b_{v}}{O(-v t)}
$$

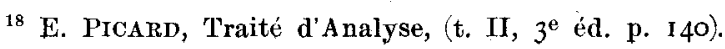


à condition qu'on ait

$$
O(v t) \neq 0
$$

pour toute valeur entière, positive, négative ou nulle, de $y$. La solution de l'équation ( $\mathrm{l}$ ) sera ainsi

$$
F(x)=\sum_{v=0}^{x}\left[\begin{array}{c}
a_{r} \\
\partial(v t)
\end{array} X^{v}+\frac{b_{v}}{\partial(-v t) X^{v}}\right]
$$

et une limitation de $O(v t)$, analogue à celle faite au $\S 17$, nous montre que cette série est uniformément convergente, pourvu que la série (91) le soit.

Supposons maintenant qu'il y ait une valeur de l'entier $v$ pour laquelle la condition (93) ne soit pas remplie; on voit facilement que l'on a dans ce cas

$$
\underset{p}{\stackrel{x}{E}} e^{v t x}=e^{r t x} O(v t)=\mathrm{o}
$$

de sorte que si, par un moyen quelconque, on a trouvé une solution périodique $f(x)$ de l'équation fonctionnelle (I), la solution générale en sera de la forme

$$
F(x)=f(x)+A e^{r t x}
$$

$A$ étant une constante.

On peut ainsi énoncer la propriété suivante:

Etant donnée l'équation fonctionnelle (I), dans laquelle $g(x)$ désigne une fonction entière, admettant la période $i \omega(\omega>0)$, cette équation admet en général une solution unique, fonction entière et admettant la même période, s'il n'y a aucun entier, positif, négatif ou nul, satisfaisant à la relation

$$
o\left(\frac{2 \pi v}{\omega}\right)=o
$$

Dans le cas contraire, il y en a une infinité, obtenues en ajoutant à une solution particulière la fonction $A e^{\frac{2 v \pi x}{\omega}}$

23. On peut ramener quelquefois la résolution de l'équation fonctionnelle (I) à celle d'une équation intégrale. Naturellement, la solution n'en sera pas unique mais, sachant résoudre l'équation intégrale obtenue, on en déduira une solution de l'équation fonctionnelle qui a servi pour point de départ. 
Supposons connues les quantités $F^{(i)}(\mathrm{o})=\lambda_{i}(i=0, \mathrm{I}, 2, \ldots m)$ et cherchons une solution de l'équation fonctionnelle ( $\mathrm{I}$ ) ayant des dérivées continues jusqu'à un certain ordre $m+\mathrm{I}$.

De la formule de Taylor (46) on déduit

$$
\begin{aligned}
F\left(x+\omega_{i}\right)=\lambda_{0}+\frac{\left(x+\omega_{i}\right)}{1 !} \lambda_{1}+\frac{\left(x+\omega_{i}\right)^{2}}{2 !} \lambda_{2}+\cdots+\frac{\left(x+\omega_{i}\right)^{m}}{m !} \lambda_{m}+ & \\
+ & \int_{0}^{x+\omega_{i}} \frac{\left(x+\omega_{i}-s\right)^{m}}{m !} F^{(m+1)}(s) d s
\end{aligned}
$$

de sorte que l'équation fonctionnelle (I) devient

$$
B_{0} \lambda_{0}+\lambda_{1} \underset{p}{E} \frac{x}{1 !}+\lambda_{2} \underset{p}{E} \frac{x^{2}}{2 !}+\cdots+\lambda_{m} \underset{p}{E} \frac{x}{m !}+\sum_{i=0}^{p} A_{i} \int_{0}^{x+\omega_{i}} \frac{\left(x+\omega_{i}-s\right)^{m}}{m !} F^{(m+1)}(s) d s=g(x)
$$

ou encore, vu les relations (22),

$$
\sum_{i=0}^{p} A_{i} \int_{0}^{x+\omega_{i}} \frac{\left(x+\omega_{i}-s\right)^{m}}{m !} F^{(m+1)}(s) d s=g(x)-\sum_{j=k}^{n} \lambda_{j} \bar{G}_{j}^{k}(x)=f(x)
$$

qui est une équation intégrale du type de M. Volterra, dont le noyau possède une ligne de discontinuité dans le domaine d'intégration.

On peut écrire encore cette équation

$$
\int_{0}^{x} \sum_{i=0}^{p} A_{i} \frac{\left(x+\omega_{i}-s\right)^{m}}{m !} F^{(m+1)}(s) d s+\sum_{i=1}^{p} A_{i} \int_{x}^{x+\omega_{i}} \frac{\left(x+\omega_{i}-s\right)^{m}}{m !} F^{(m+1)}(s) d s=f(x)
$$

ou encore, vu la relation (22)

(97) $\int_{0}^{x} \bar{G}_{m}^{k}(x-s) F^{(m+1)}(s) d s+\sum_{i=1}^{p} A_{i} \int_{x}^{x+\omega_{i}} \frac{\left(x+\omega_{i}-s\right)^{m}}{m !} F^{(m+1)}(s) d s=f(x)$.

Pour résoudre cette équation intégrale, nous allons y introduire un paramètre $\lambda$, de sorte qu'on pourra écrire 


$$
\int_{0}^{x} \overline{G_{m}^{k}}(x-s) \boldsymbol{\Phi}(s) d s+\lambda \sum_{i=1}^{p} \int_{i}^{x+\omega_{i}} \begin{gathered}
\left(x+\omega_{i}-s\right)^{m} \\
m !
\end{gathered}
$$

avec $F^{(m+1)}(x)=\Phi(x)$.

Si nous cherchons à satisfaire formellement à cette équation intégrale par une série en $\lambda$

$$
\Phi(x)=\Phi_{0}(x)+\lambda \Phi_{1}(x)+\lambda^{2} \Phi_{z}(x)+
$$

nous sommes conduits à la résolution d'une infinité d'équations intégrales de M. Volterra, de première espèce

$$
\begin{gathered}
\int_{i=}^{k} \bar{G}_{m}^{k}(x-s) \boldsymbol{\Phi}_{0}(s) d s=f(x) \\
\int_{1}^{x} G_{m}^{k}(x-s) \boldsymbol{\Phi}_{n}(s) d s=-\sum_{i=1}^{p} A_{i} \int_{x}^{x+c_{i}} \frac{\left(x+\omega_{i}-s\right)^{m}}{m !} \boldsymbol{\Phi}_{n-1}(s) d s \\
n=1,2, \ldots
\end{gathered}
$$

Or ces équations sont résolubles, puisque $G_{n}^{k}(x-x)=\bar{G}_{n}^{k}(0)=\frac{B_{n}}{n !} \neq 0$, mais elles n'admettent pas en général des solutions uniques, puisque les seconds membres ne sont pas nuls pour $x=0$, comme l'exige la théorie de M. Volterra, à ce qu'on s'attendait, comme nous l'avons déjà remarqué, puisque la solution de l'équation fonctionnelle $(\mathrm{I}) \mathrm{n}$ 'est pas entièrement déterminée par la connaissance des $(m+\mathrm{I})$ quantités $\lambda_{i}$. Cependant, on peut résoudre ces équations intégrales et de leurs solutions on déduira une solution de l'équation fonctionnelle ( $\mathrm{I}$ ).

La résolution des équations intégrales (1OO) et (IOI) se ramène facilement à celle d'une infinité d'équations différentielles linéaires, puisque le noyau commun $\bar{G}_{m}^{k}(x-s)$ des équations intégrales considérées est un polynome: On n'a qu'à intégrer par parties dans les premiers membres et à poser après

$$
\int_{0}^{x} \Phi_{n}(x) d x=\varphi_{n}(x)
$$

pour arriver à ces équations différentielles. 
24. Considérons enfin l'équation fonctionnelle suivante

$$
\varphi_{0}(x) F(x)+\varphi_{1}(x) F\left(x+\omega_{1}\right)+\cdots+\varphi_{p}(x) F\left(x+\omega_{p}\right)=g(x)
$$

avec les mêmes hypothèses sur les écarts $\omega_{i} ; g(x)$ sera censée satisfaire à la condition (74), tandis que nous supposerons les fonctions $\varphi_{i}(x)$ telles que

$$
\lim _{x=\infty} \varphi_{i}(x)=A_{i}
$$

$A_{i}$ étant des constantes.

Posons

$$
\varphi_{i}(x)=A_{i}+\psi_{i}(x), \quad \lim _{x=\infty} \psi_{i}(x)=0
$$

Pour résoudre l'équation fonctionnelle (IO2), nous allons introduire un paramètre $\lambda$ en l'écrivant

$$
\underset{p}{\stackrel{x}{E}} F(x)=g(x)+\lambda\left[\psi_{0}(x) F(x)+\psi_{1}(x) F\left(x+\omega_{1}\right)+\cdots+\psi_{p}(x) F\left(x+\omega_{p}\right)\right]
$$

et en cherchant à la satisfaire par un développement formel en série suivant les puissances de $\lambda$

$$
F(x)=F_{0}(x)+\lambda F_{1}(x)+\lambda^{2} F_{2}^{\prime}(x)+\cdots .
$$

Nous obtenons les équations d'approximation

$$
\underset{p}{E} F_{0}(\dot{x})=g(x)
$$

$$
\underset{p}{E} F_{1}(x)=\psi_{0}(x) F_{0}(x)+\psi_{1} F_{0}\left(x+\omega_{1}\right)+\cdots+\psi_{p}(x) F_{0}\left(x+\omega_{p}\right)
$$

et en général

$$
\underset{p}{\stackrel{x}{E}} \boldsymbol{F}_{n}(x)=g_{n}(x)
$$

avec

$(108) \quad g_{n}(x)=\psi_{0}(x) F_{n-1}(x)+\psi_{1}(x) F_{n-1}\left(x+\omega_{1}\right)+\cdots+\psi_{p}(x) F_{n-1}\left(x+\omega_{p}\right)$

La première équation, qui donne $F_{0}(x)$, n'est autre que l'équation fonctionnelle (I), que nous venons d'étudier; sa solution est donnée, par exemple, par la relation $(67)$

36-33617. Acta mathematica. 62. Imprimé le 17 janvier 1934 


$$
\boldsymbol{F}_{\mathbf{0}}(x+h)=\sum_{j=0}^{m+k} G_{j}^{k}(h) f^{(j)}(x)+\int_{0}^{\infty} \dot{G}_{m+k}^{k}(h-t) g^{(m+1)}(x+t) d t
$$

avec $f^{(k)}(x)=g(x)$. On en obtient ainsi la solution principale.

- Passons ensuite à l'équation (I06): elle est de la même forme que (I); on n'a qu'à y remplacer $g(x)$ par $g_{1}(x)$, donnée par (IO8) à l'aide de l'expression trouvée pour $F_{0}(x)$; on a

$$
F_{1}(x+h)=\sum_{j=0}^{m+k} G_{j}^{k}(h) f_{2}^{(j)}(x)+\int_{0}^{\infty} \dot{G}_{m+k}^{k}(h-t) g_{1}^{(m+1)}(x+t) d t
$$

qui est valable lorsque $g_{1}(x)$ admet une dérivée d'ordre $(m+1)$ qui reste continue à partir d'une certaine valeur $a$ de $x$ et qui satisfait à la condition

$$
\lim _{x=\infty} x^{1+\alpha} g_{1}^{m+1\rangle}(x)=0 .
$$

Cette condition est remplie si les $\psi_{i}(x)$ admettent des dérivées de l'ordre $(m+\mathrm{I})$, continues pour $x \geq a$ et si les produits $x^{r+1} \psi_{i}^{(v)}(x)(v=0, \mathrm{I}, 2, \ldots m+1)$ restent bornés quand $x$ croît indéfiniment, ce qui arrive, par exemple, lorsque les $\psi_{i}(x)$ sont holomorphes autour du point à l'infini et nulles en ce point.

Or, d'après les propriétés de la solution principale $F_{0}(x)$, on déduit qu'elle admet des dérivées continues jusqu'au $(m+1)^{\text {e }}$ ordre, satisfaisant aux conditions

$$
\lim \frac{F_{v}^{(m+1-v)}(x)}{x^{v+1}}=0, \quad \nu=0, \mathrm{I}, 2, \ldots m+\mathrm{I} .
$$

D'autre part

$$
\begin{aligned}
g_{1}^{(m+1)}(x) & =\sum_{i=0}^{p}\left[\psi_{i}(x) F_{0}\left(x+\omega_{i}\right)\right]^{(m+1)} \\
& =\sum_{i=0}^{p} \sum_{r=0}^{m+1}\left(\begin{array}{c}
m+1 \\
r
\end{array}\right) \psi_{i}^{(r)} F_{o}^{(m+1-r)}\left(x+\omega_{i}\right)
\end{aligned}
$$

done

$$
\lim _{x=\infty} g_{1}^{(m+1)}(x)=\lim _{x=\infty} \sum_{i=0}^{p} \sum_{r=0}^{m+1}\left(\begin{array}{c}
m+1 \\
r
\end{array}\right)\left(x^{r+1} \psi_{i}^{(r)}\right)\left(\frac{F_{m}^{(m+1-r)}\left(x+\omega_{i}\right)}{x^{r+1}}\right)=0
$$

Un calcul analogue nous aurait conduit, en resserant de plus les hypothèses sur $g^{(m+1)}(x)$, à la limite plus précise 


$$
\lim _{x=\infty} x^{1+\alpha} g_{1}^{(m+1)}(x)=0
$$

et on légitime ainsi l'expression (I IO), donnée pour la seconde approximation $F_{1}(x+h)$.

L'algorithme par lequel on passe de la fonction $F_{0}(x)$ à $F_{1}(x)$ peut être itéré pour passer de $F_{1}(x)$ à $F_{2}(x)$ et ainsi de suite. On détermine ainsi, de proche en proche, les approximations successives $\boldsymbol{F}_{n}(x+h)$, qui nous donnent la solution principale cherchée de l'équation fonctionnelle (IO2)

$$
F(x+h)=F_{0}(x+h)+\lambda F_{1}(x+h)+\lambda^{2} F_{2}(x+h)+\cdots
$$

le développement étant uniformément et absolument convergent pour $\lambda$ suffisament petit lorsque $x$ reste compris dans un intervalle fini quelconque $(a, b)$, c'est ce qu'on démontre aisément en majorant les diverses approximations $\boldsymbol{F}_{\boldsymbol{n}}(x+h)$.

Ce problème a été traité aussi par M. R. Raclis (loc. cit. 7) dans le cas particulier où l'opérateur général $\underset{p}{\stackrel{x}{E}}$ se réduit à

$$
\stackrel{p, q}{\stackrel{p}{\alpha_{1} \alpha_{2} \cdots \alpha_{p} \beta_{1} \beta_{2}} \cdots \beta_{q}}
$$

\section{Systèmes d'équations fonctionnelles.}

25. Ce qui précède permet la résolution, dans certains cas, des systèmes linéaires d'équations aux différences finies.

Nous allons en donner quelques exemples.

Considérons le système d'équations fonctionnelles

$$
\begin{gathered}
\underset{p}{E} F_{i}(x)+a_{i 1} F_{1}(x)+a_{i 2} F_{2}(x)+\cdots+a_{i p} F_{p}(x)=g_{i}(x) \\
i=\mathrm{I}, 2, \ldots p,
\end{gathered}
$$

$\stackrel{x}{E}$ ayant la même signification que dans l'équation fonctionnelle (I), tandisque les $a_{i j}$ sont des constantes, à déterminant non nul

$$
D_{p}=\left|a_{i 1} a_{i 2} \cdots a_{i p}\right| \neq 0 .
$$

On peut résoudre le système (II3) encore par la méthode des approxima- 
tions successives. Cherchons en effet, à y satisfaire par des développements de la forme

$$
\boldsymbol{F}_{i}(x)=\boldsymbol{F}_{i 0}(x)+\lambda \boldsymbol{F}_{i 1}(x)+\lambda^{2} \boldsymbol{F}_{i 2}(x)+
$$

après avoir introduit le paramètre $\lambda$ dans les équations (113), ce qui permet de les écrire

$$
\underset{p}{\stackrel{x}{E}} F_{i}(x)+\lambda\left[a_{i 1} F_{1}(x)+a_{i 2} F_{2}(x)+\cdots+a_{i p} F_{p}(x)\right]=g_{i}(x)
$$

Nous obtenons, pour les premières approximations $F_{i 0}(x)$, les équations fonctionnelles

$$
\underset{p}{\boldsymbol{E}} \boldsymbol{F}_{i 0}(x)=g_{i}(x)
$$

qui sont de la forme (I) et dont les solutions principales sont données par (67)

$$
\begin{gathered}
F_{i 0}(x+h)=\sum_{j=0}^{m+k} G_{j}^{k}(h) f_{i}^{(j)}(x)+\int_{0}^{\infty} \dot{G}_{m+k}^{k}(h-t) g_{i}^{(m+1)}(x+t) d t \\
i=1,2, \ldots p
\end{gathered}
$$

solutions valables dans les mêmes conditions que pour l'équation fonctionnelle ( $\mathrm{I}$ ), c'est-à-dire, ayant une dérivée continue de l'ordre $(m+I)$ et, en ce qui concerne les fonctions données $g_{i}(x)$, devant satisfaire à la condition (74).

Une fois les fonctions $F_{i 0}(x)$ déterminées, on passe aux $F_{i 1}(x)$, qui sont données par les équations

$$
\underset{p}{\stackrel{x}{E} F_{i 1}(x)}=-\left[a_{i 1} F_{10}(x)+a_{i 2} F_{20}(x)+\cdots+a_{i p} F_{p 0}(x)\right]
$$

également de la même forme que (I) et dont les solutions principales s'écrivent d'après la relation (67); en effet, les seconds membres satisfont aux mêmes conditions que ceux des équations (I 16), vu les propriétés des fonctions déjà déterminées, $F_{i 0}(x)$.

De cette manière, on obtient de proche en proche les approximations successives $F_{i n}(x)$, qui conduisent à la solution principale du système d'équations fonctionnelles proposé (I I 5), représentée par les développements 
( I 19)

$$
F_{i}(x+h)=F_{i 0}(x+h)+\lambda F_{i 1}(x+h)+\lambda^{2} F_{i 2}(x+h)+\cdots
$$

dont la convergence absolue et uniforme s'établit, pour les petites valeurs de $\lambda$, en majorant les diverses approximations $F_{\text {in }}(x)$.

26. On peut trouver, toujours à l'aide des approximations successives, une solution $d u$ système d'équations fonctionnelles par un procédé qui n'exige aucune intégration, finie ou non.

Désignons par $A_{i j}$ le mineur correspondant à l'élément $a_{i j}$ dans le déterminant $D_{p}$ et multiplions les deux membres des équations fonctionnelles (I i 3 ) respectivement par $A_{i j}, j$ étant fixe et en faisant varier $i$ de I à $p$. En posant

$$
G_{j}(x)=\sum_{i=1}^{p} A_{i j} g_{i}(x)
$$

le système (I I3) se transforme dans le suivant

$$
\begin{gathered}
\left.\underset{p}{\stackrel{x}{E}\left[A_{1 j} F_{1}(x)+A_{2 j} F_{2}(x)\right.}+\cdots+A_{p j} F_{p}(x)\right]+D_{p} F_{j}(x)=G_{j}(x) \\
j=\mathbf{I}, 2, \ldots p
\end{gathered}
$$

que nous écrirons encore, en $\mathrm{y}$ introduisant un paramètre $\lambda$

$$
\begin{gathered}
D_{p} F_{j}(x)=G_{j}(x)+\lambda \underset{p}{\stackrel{x}{E}}\left[A_{1 j} F_{1}(x)+A_{2 j} F_{2}(x)+\cdots+A_{p j} F_{p}(x)\right] \\
j=1,2, \ldots p .
\end{gathered}
$$

Si nous essayons à y satisfaire par des développements en série de $\lambda$

$$
F_{j}(x)=F_{j 0}(x)+\lambda F_{j 1}(x)+\lambda^{2} F_{j 2}(x)+\cdots
$$

nous obtenons comme premières approximations

$$
D_{p} F_{j 0}(x)=G_{j}(x)
$$

on aura ensuite

$$
\begin{gathered}
D_{p} F_{j 1}(x)=\underset{p}{E}\left[A_{1 j} F_{10}(x)+A_{2 j} F_{20}(x)+\cdots+A_{p j} F_{p 0}(x)\right] \\
j=\mathrm{I}, 2, \ldots p
\end{gathered}
$$

ou encore

$$
D_{p}^{2} F_{j 1}(x)=\underset{p}{\stackrel{x}{E}}\left[A_{1 j} G_{1}(x)+A_{2 j} G_{2}(x)+\cdots+A_{p j} G_{p}(x)\right]
$$


En général,

$$
D_{p} F_{j n}(x)=\underset{p}{\underset{E}{E}}\left[A_{1 j} F_{1, n-1}^{\prime}(x)+A_{2 j} F_{2, n-1}(x)+\cdots+A_{p j} F_{p, n-1}^{\prime}(x)\right] .
$$

Supposons les fonctions $y_{i}(x)$ décroîssantes, à partir d'une certaine valeur de $x, x \geq a$, de manière qu'elles tendent vers zéro, lorsque $x$ croît indéfiniment; on pourra donc écrire, pour $x \geq a$

$$
\left|g_{i}(x)\right|<M
$$

$M$ étant la plus grande valeur absolue des diverses fonctions $g_{i}(x)$; désignons encore par $A$ la plus grande valeur absolue des constantes $A_{i j}, A_{i}$; on aura

$$
\left|D_{p}\right|\left|F_{j 0}(x)\right|=\mid\left(\dot{x}_{j}(x) \mid<p A M .\right.
$$

On aura ensuite

$$
\left|\underset{p}{E} A_{i j} G_{i}(x)\right|<A \sum_{0}^{p}\left|A_{i} G_{i}\left(x+\omega_{v}\right)\right|<(p+\mathrm{I}) A^{2} M
$$

donc

$$
D_{p}^{2}\left|F_{j 1}(x)\right|<(p+\mathrm{I}) A(p A M)
$$

on obtient de la même manière

$$
\left|D_{p}^{3}\right|\left|F_{j 2}(x)\right|<(p+1)^{2} A^{2}(p A M)
$$

et en général

$$
\left|D_{p}^{n+1}\right|\left|F_{j n}(x)\right|<A^{n}(p+1)^{n}(p A M) .
$$

Les développéments (I 22) des fonctions $F_{j}^{\prime}(x)$ convergent, par suite, si la série

$$
\sum_{0}^{\infty} \frac{|\lambda|^{n} A^{n}(p+1)^{n}}{\left|D_{p}\right|^{n}}
$$

converge, ce qui a lieu si

$$
\text { (123) } \quad|\hat{\lambda}|<\frac{\left|D_{p}\right|}{(p+\mathrm{I}) A} .
$$

27. Ces considérations peuvent être appliquées à des systèmes plus géné-

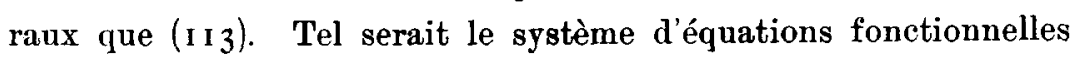


( 124$)$

$$
\begin{gathered}
\underset{p_{i}}{E} F_{j}(x)+a_{j 1} F_{1}(x)+a_{j 2} F_{2}(x)+\cdots+a_{j p_{i}} F_{p_{i}}(x)=g_{j}(x) \\
j=\mathrm{I}, 2, \ldots p_{i}
\end{gathered}
$$

les $p_{i}$ étant des entiers positifs, différents ou même égaux (quelquesuns), en nombre égal au plus grand d'entre eux.

De même, le système plus général

$$
\begin{gathered}
\varphi_{i 0}(x) F_{i}(x)+\varphi_{i 1} F_{i}\left(x+\omega_{1}\right)+\cdots \varphi_{i p} F_{i}\left(x+\omega_{p}\right)+ \\
+\Phi_{i 1}(x) F_{1}(x)+\Phi_{i 2}(x) F_{2}(x)+\cdots+\boldsymbol{\Phi}_{i p}(x) F_{p}(x)=g_{i}(x) \\
i=\mathrm{I}, 2, \ldots p
\end{gathered}
$$

dans lequel on a

$$
\lim _{x=\infty} \varphi_{i j}(x)=A_{j}, \quad \lim _{x=\infty} \boldsymbol{\Phi}_{i j}(x)=a_{i j}
$$

se traite par les mêmes procédés, employés pour une seule équation. 